\title{
THE SEARCH FOR, AND DISCOVERY OF, THE HIGGS BOSON AT CMS
}

\author{
DANIEL GREEN \\ Fermilab, P.O. Box 500, Batavia, IL 60510, USA \\ dgreen@fnal.gov
}

Received 8 December 2012

Accepted 10 December 2012

Published 18 January 2013

\begin{abstract}
The Higgs field was first proposed almost 50 years ago. Twenty years ago the tools needed to discover the Higgs boson, the large hadron collider and the CMS and ATLAS experiments, were initiated. Data taking was begun in 2010 and culminated in the announcement of the discovery of a "Higgs-like" boson on 4 July 2012. This discovery completes the Standard Model (SM) of high energy physics, if it is indeed the hypothesized SM Higgs particle. Future data taking will explore the properties of the new $125 \mathrm{GeV}$ particle to see if it has all the attributes of an SM Higgs and to explore the mechanism that maintains its "low" mass.
\end{abstract}

Keywords: Higgs boson; di-photon; four-lepton.

PACS numbers: 14.80.Bn, 13.85.Rm

\section{Contents}

1. Introduction and History . . . . . . . . . . . . . . . . . . . . . . 2

2. Theory of the Standard Model Higgs Boson . . . . . . . . . . . . . . 2

3. The LHC Accelerator . . . . . . . . . . . . . . . . . . . . . . . . 5

4. The CMS Detector and the Higgs . . . . . . . . . . . . . . . . . . . 8

5. The CMS Detector and Analysis - Overview . . . . . . . . . . . . . 12

6. Cosmic Ray Data Taking and Other Preparations in CMS . . . . . . . . 17

7. The 2010 Start of $7 \mathrm{TeV}$ Data Taking . . . . . . . . . . . . . . . . . . . 19

8. The 2011 Data Taking at $7 \mathrm{TeV}$ - Higgs Hunt Hints . . . . . . . . . . 22

9. Data Taking in 2012 - The Higgs Discovery . . . . . . . . . . . . . . 25

10. The Future . . . . . . . . . . . . . . . . . . . . . . . . . . . . 36

11. Conclusion . . . . . . . . . . . . . . . . . . . . . . . . . . 47

Acknowledgments . . . . . . . . . . . . . . . . . . . . . . . . . . . . 47

References . . . . . . . . . . . . . . . . . . . . . . . . . 48

"Nothing is too wonderful to be true" — Michael Faraday "Then felt I like some watcher of the skies when a new planet swims into his ken ... Look'd ... with a wild surmise" — John Keats 


\section{Introduction and History}

The SM of particle physics explains a very wide range of phenomena in terms of spin $\frac{1}{2}$ quarks and leptons as the building blocks of matter; matter which interacts by exchanging the spin 1 quanta of the strong, electromagnetic and weak forces, the gluons, photons and $W / Z$ bosons, respectively. However, the SM by itself requires that the force carriers be massless in order to preserve the gauge symmetry, a symmetry which elegantly predicts the existence of these force carriers as a consequence of that principle. Indeed the photons and gluons are massless as is the hypothesized graviton due to a very similar requirement on the gravitational force. The problem is that the $W$ and $Z$ of the weak interactions are heavy, weighing $80 \mathrm{GeV}$ and $91 \mathrm{GeV}$, respectively. Those masses break the symmetry of the weak interactions; electroweak symmetry breaking (EWSB).

A solution to the problem in superconductivity of the exclusion of a magnetic field in a superconductor was invoked by Landau and Ginzberg ${ }^{1}$ in 1950. In their formulation, a vacuum field gave mass to the photons inside a superconductor, therefore excluding them due to a Yukawa-like suppression of the field. The Higgs field is postulated to be constant throughout the Universe in space and time and which, by interacting with the $W$ and $Z$, gives them their mass in direct analogy to the hypothesis of Landau and Ginzberg. ${ }^{2-7}$ In addition, it is also postulated that a Yukawa coupling of the matter particles to the Higgs field also gives them a mass, albeit with unspecified coupling constants. Although the weak interactions appear to be weak at low energies, when the energy is comparable to the $W$ and $Z$ masses, it is observed that the basic weak coupling constant is comparable to that of the electromagnetic interactions. In that sense, electromagnetism and the weak interactions are then unified. ${ }^{8-10}$

The Higgs field itself is not directly observable, but the Higgs quantum, the Higgs boson, can be excited from the Higgs vacuum field. That boson can then be observed given the necessary tools, an accelerator operating at a sufficiently high energy and $p-p$ collision rate and detectors sufficiently complex to observe the decay products of the Higgs quantum in the complex and hostile environment which exists at a high luminosity $p-p$ collider. These two indispensable tools needed many years of development in parallel before a decisive search for the Higgs boson could be enabled.

\section{Theory of the Standard Model Higgs Boson}

The SM is a compact description of all nongravitational physics as illustrated in Fig. 1. The matter particles are the six strongly interacting quarks, the three charged, electromagnetically interacting leptons and the three neutral weakly interacting neutrinos. The force carriers are the photon (electromagnetic), the gluon (strong) and the $W$ and $Z$ (weak). The charm quark and the tau lepton were discovered at SLAC in the 1970s. The $W$ and $Z$ were discovered at CERN in the 1980s.

The $b$ and top quark were discovered at Fermilab, the latter in the 1990s. Finally, 


\section{THE STANDARD MODEL}

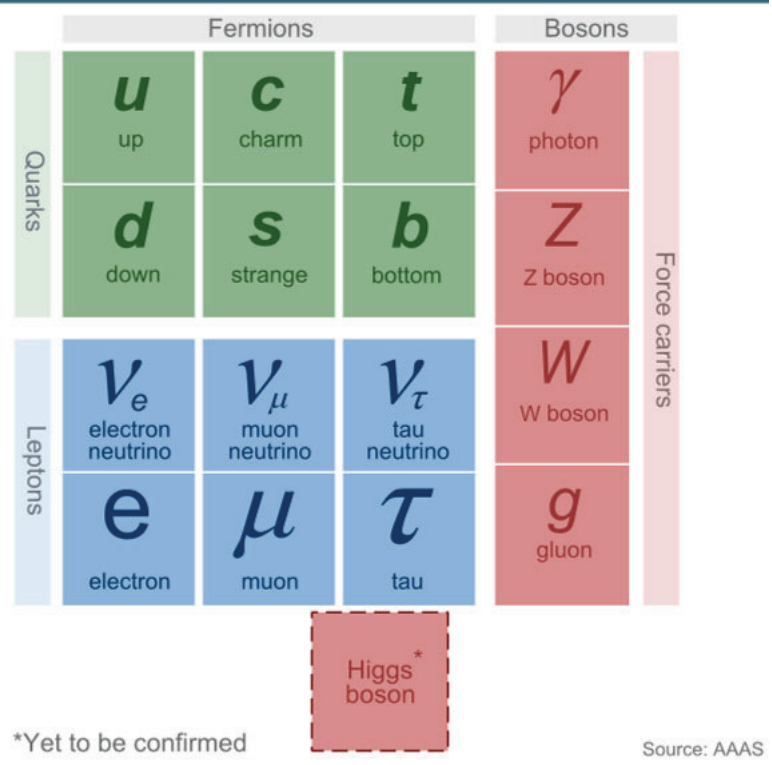

Fig. 1. (Color online) The particles comprising the SM. The dots indicate where the particles were discovered, blue $=\mathrm{SLAC}$, yellow $=$ FNAL and red $=$ CERN .

the tau neutrino was observed at Fermilab in 2000. All these particles which define the "periodic table" of the SM were discovered subsequent to the formulation of the Higgs hypothesis.

The Higgs field is assumed to be a scalar, represented by $\phi$, where $P$ is the momentum, $M$ is the boson mass and $S$ is the action. The Lagrangian density, $\ell$, for the Higgs is shown in Eq. (1). Since the action is dimensionless, the dimension of the Higgs field is that of mass

$$
\ell=\bar{\phi}\left(P^{2}-M^{2}\right) \phi, \quad S=\int \ell d^{4} x, \quad[S]=1, \quad[\phi]=M .
$$

The self-interactions of the Higgs field are postulated by defining a potential, Eq. (2) which contains two unknown parameters and which contains a quartic self-coupling term. The field can have a nonzero minimum, giving the Higgs field a nonzero vacuum expectation value, $\langle\phi\rangle$

$$
V=\mu^{2} \phi^{2}+\lambda \phi^{4}, \quad\langle\phi\rangle-\frac{\mu^{2}}{2 \lambda}, \quad V(\langle\phi\rangle)=-\lambda\langle\phi\rangle^{4} .
$$

The Higgs boson is the excitation of the Higgs field $\phi_{H}$. Expanding the field about the minimum vacuum value the potential experienced by the Higgs boson (Eq. (3)), has four terms:

$$
\phi=\langle\phi\rangle+\phi_{H}, \quad V(\phi)=-\lambda\langle\phi\rangle^{4}+4 \lambda\langle\phi\rangle^{2} \phi_{H}^{2}+4 \lambda\langle\phi\rangle \phi_{H}^{3}+\lambda \phi_{H}^{4} .
$$


By inspection (Eq. (1)), the Higgs acquires a mass (Eq. (4)), and has triple and quartic self-couplings. There is also a nonzero "cosmological term" which will be mentioned later in the context of dark energy. The mass is proportional to the vacuum field value but with an unknown coefficient

$$
M_{H}=2 \sqrt{\lambda}\langle\phi\rangle .
$$

The gauge replacement of the ordinary derivative by the covariant derivative is familiar from classical electromagnetism. Applying that replacement in Eq. (5) to the Higgs Lagrangian kinetic energy term with the electroweak bosons in the covariant fields leads to masses for the $W$ and $Z$ while keeping the photon massless, which was the goal of the Higgs hypothesis. The vector force carriers are represented by $\varphi$

$$
\begin{gathered}
\partial \rightarrow D=\partial-i g V, \quad V=W, Z, \gamma, \\
D \bar{\phi} D \phi \sim\left(\frac{g_{W}^{2}}{2}\right) \bar{\varphi}_{W} \varphi_{W}\left(\langle\phi\rangle+\phi_{H}\right)\left(\langle\phi\rangle+\phi_{H}\right), \\
M_{W}=\frac{g_{W}\langle\phi\rangle}{\sqrt{2}}, \quad M_{Z}=\frac{M_{W}}{\cos \theta_{W}}, \quad M_{\gamma}=0 .
\end{gathered}
$$

The vacuum Higgs field gives mass to the $W$ and $Z$. The Higgs boson then must (Eq. (6)), interact with the $W$ in triple and quartic couplings. These couplings are completely specified in the SM

$$
\ell_{H}=\frac{\alpha_{W}\langle\phi\rangle}{2\left[\phi_{H} \varphi_{W} \varphi_{W}\right]}+\frac{\alpha_{W}}{2\left[\phi_{H} \phi_{H} \varphi_{W} \varphi_{W}\right]} .
$$

Although it is not strictly necessary, a Yukawa coupling of the Higgs field to the fermions, $\psi$ can be postulated. In analogy to the bosons the fermions then acquire a mass proportional to the Higgs vacuum field, Eq. (7), and possess coupling to the Higgs boson whose strength is proportional to the fermion's mass. However, this scheme is ad hoc and is not necessary in the SM save for the appealing simplicity and economy of the scheme

$$
\begin{aligned}
& \ell_{f}=g_{f}[\bar{\psi} \phi \psi], \text { Dirac, } \bar{\psi}(\not \partial-m) \psi, \quad m_{f}=g_{f}\langle\phi\rangle, \\
& \ell_{f}=m_{f} \bar{\psi} \psi+g_{f}\left[\bar{\psi} \phi_{H} \psi\right], \quad g_{f}=g_{W}\left(m_{f} / M_{W}\right) / \sqrt{2} .
\end{aligned}
$$

Numerically, the Fermi coupling constant, $G$, is measured in muon decay and the weak coupling constant in neutrino interactions. The data acquired for muon decay, Eq. (8), allows for the determination of the Higgs vacuum field. Therefore, there is only one unknown parameter remaining in the SM and that would be determined by measuring the Higgs mass

$$
\frac{G}{\sqrt{2}}=\frac{g_{W}^{2}}{8 M_{W}^{2}}, \quad \frac{M_{W}}{g_{W}}=\frac{\langle\phi\rangle}{\sqrt{2}}, \quad\langle\phi\rangle=\frac{\sqrt{2}}{4 G}, \quad\langle\phi\rangle=174 \mathrm{GeV} .
$$

The weak coupling constant, $g_{W}$, the Weinberg angle relating the electromagnetic coupling and the weak coupling, $\sin \theta_{W}$, the weak constant, $\alpha_{W}$, and the masses 
predicted for the $W$ and $Z$, Eq. (9), using the vacuum field from Eq. (8) were spectacularly confirmed at CERN in the 1980s. The Higgs boson mass is not predicted, but looking ahead and taking $125 \mathrm{GeV}$ the remaining unknown parameter of the Higgs potential is determined. Therefore, if the Higgs mass is known, then in the SM all phenomena can be predicted

$$
\begin{aligned}
& \sin \theta_{W}=0.481, \quad g_{W}=0.63, \quad \alpha_{W}=\frac{1}{31.6}, \quad M_{W}=80 \mathrm{GeV}, \\
& M_{Z}=91 \mathrm{GeV}, \quad M_{H}=125 \mathrm{GeV} \rightarrow \lambda=0.60, \quad \mu=191 \mathrm{GeV} .
\end{aligned}
$$

Nevertheless, the mass was not known when the LHC, ATLAS and CMS were being designed and therefore the designers of the accelerator and detectors needed to create instruments appropriate to a very wide range of possible Higgs masses, covering the $114 \mathrm{GeV}$ limit set at LEP, ${ }^{11,12}$, a to the approximately $1 \mathrm{TeV}$ mass set by perturbative unitarity in $W+W$ scattering. Many early estimates of Higgs production cross-sections and search strategies set out the theoretical expectations for the Higgs search. ${ }^{14,15}$

\section{The LHC Accelerator}

The progress of high energy physics over the last five decades is shown telegraphically in the "Livingston plot" of Fig. 1. The axes are the date when a new accelerator began data taking versus the approximate energy available to produce new, heavy particles at that facility. Over time that ability to reach higher masses has increased approximately exponentially. There are two trajectories indicated, one for lepton colliders where all the CM energy is available to make new particles and hadron colliders, where the fundamental quarks and gluons have a reduced "parton CM energy" available. Also shown in Fig. 2 are the masses of the quarks and bosons which were discovered during this period (Fig. 1). The allowable mass range of the Higgs boson, $114 \mathrm{GeV}$ to about $1000 \mathrm{GeV}$ is also shown and indicates that the LHC has sufficient energy to cover this mass range. Indeed, the design criteria for both the LHC CM energy and luminosity were to be able to decisively search for the Higgs boson in a few years of data taking at design luminosity.

The LHC complex uses the existing LEP tunnel at CERN. To reach the needed $1 \mathrm{TeV}$ mass scale, the LHC is designed to operate as a proton-proton collider at $14 \mathrm{TeV}$ center of mass (CM) energy. To explore the $1 \mathrm{TeV}$ mass scale in a reasonable period of time, a "luminosity" of $10^{34} / \mathrm{cm}^{2} \mathrm{~s}$ or a total reaction rate of almost a trillion collisions per second is needed. The LHC was approved in 1994, 30 years after the Higgs hypothesis was made and construction was started in 1998.

The LHC is a machine aimed at discovery and that required many new cutting edge technologies. ${ }^{16,17}$ More complete descriptions are available on the CERN web sites. ${ }^{18}$ The heart of the LHC lies in the superconducting dipole magnets. Because 


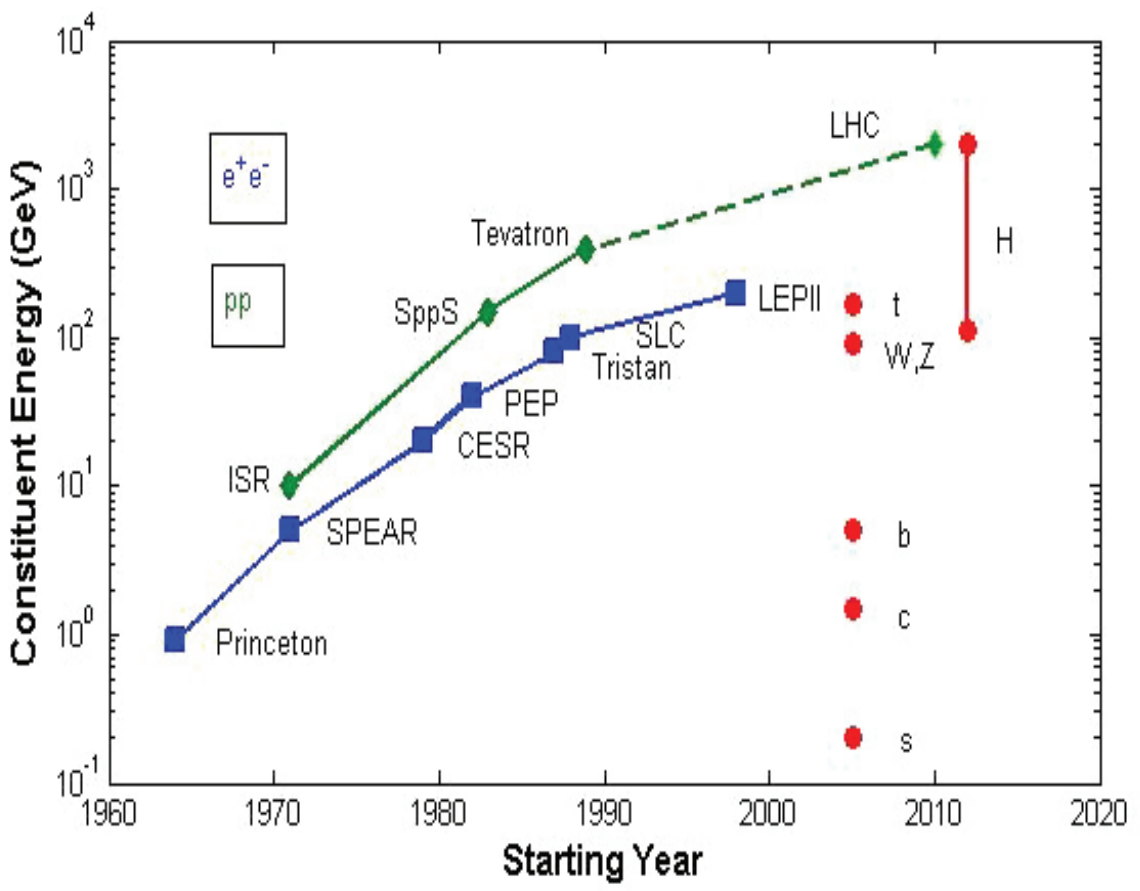

Fig. 2. Plot of the year of commissioning a new accelerator and the energy therein made available for new particle production.

the LEP tunnel was reused for the LHC the dipoles needed to be very strong in order to achieve the $14 \mathrm{TeV}$ CM energy which makes the LHC the highest energy collider in the world. The dipole field is $8.33 \mathrm{~T}$, and the magnets are operated at $1.9 \mathrm{~K}$ which means the LHC is colder than the Cosmic Microwave Background (CMB). The cryogenic plant uses $100 \mathrm{~T}$ of liquid He making it the largest in the world. The dipole current is 11,700 A at full field. The LHC required the construction, installation and commissioning of 1232 dipoles and 858 quadrupoles. The dipoles have 11 GJ of stored magnetic energy which necessitates an elaborate and reliable quench protection system. The power consumed at the CERN site is $120 \mathrm{MW}$. A view of the dipoles installed in the LHC tunnel appears in Fig. 3.

The LHC is designed to have the highest reaction rate of any collider with a design value of almost one GHz. This is accomplished by using very intense beams of protons. When operating at design luminosity there are 2808 r.f. bunches per beam, spaced by $25 \mathrm{~ns}=7.5 \mathrm{~m}$. That means there is a bunch crossing rate for collisions of about $40 \mathrm{MHz}$. Given the total proton-proton cross-section that leads to the almost $1 \mathrm{GHz}$ reaction rate. The bunch intensity is about $1.2 \times 10^{11}$ protons per bunch and each bunch is about $5 \mathrm{~cm}$ long and 16 microns wide. At full LHC energy each beam carries $362 \mathrm{MJ}$ which means the two beams have sufficient energy to melt about 1 ton of cryogenic $(2 \mathrm{~K})$ copper. 


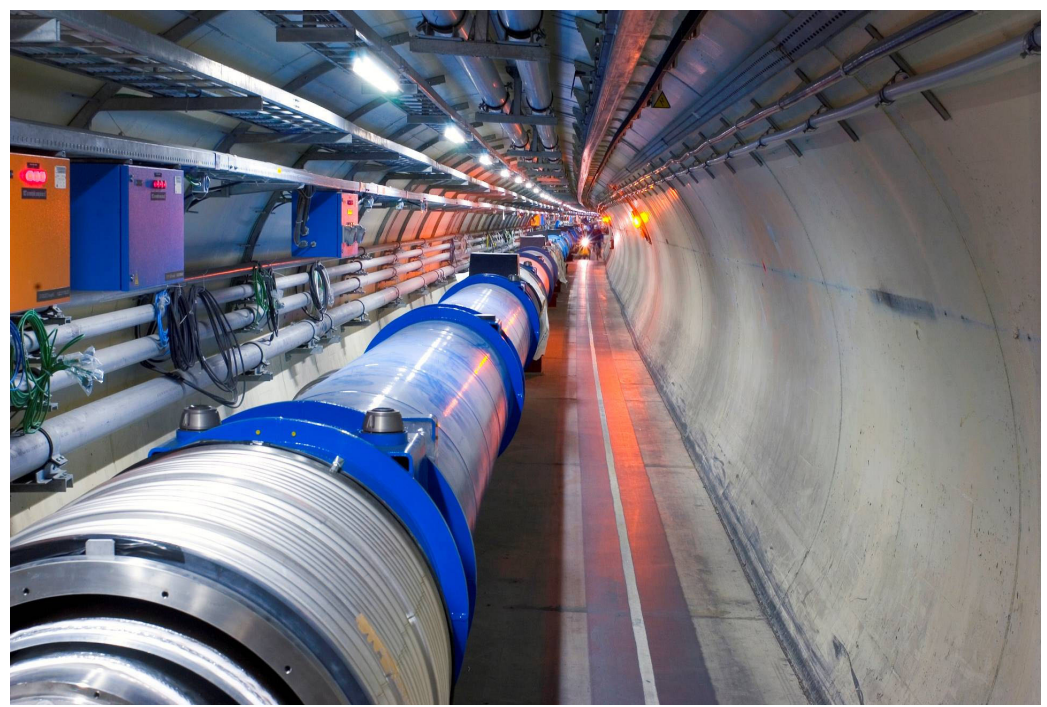

Fig. 3. Photograph of the LHC tunnel showing the superconducting dipole magnets.

Because of the large stored beam energy a precise and reliable beam position monitoring system and a complex array of passive collimators was installed and a tested beam abort and dump system was put in place and tested. A plot of the stored energy in the beams and the beam energy for several contemporary accelerators is shown in Fig. 4. Clearly the LHC stands in a unique and challenging position.

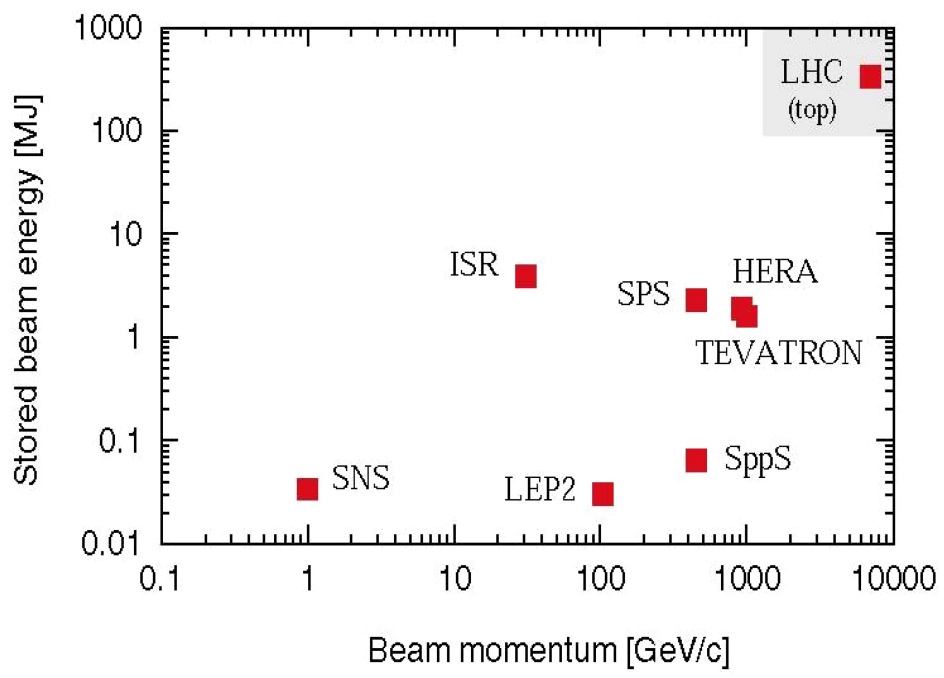

Fig. 4. Energy stored in the beams for several contemporary accelerators. 


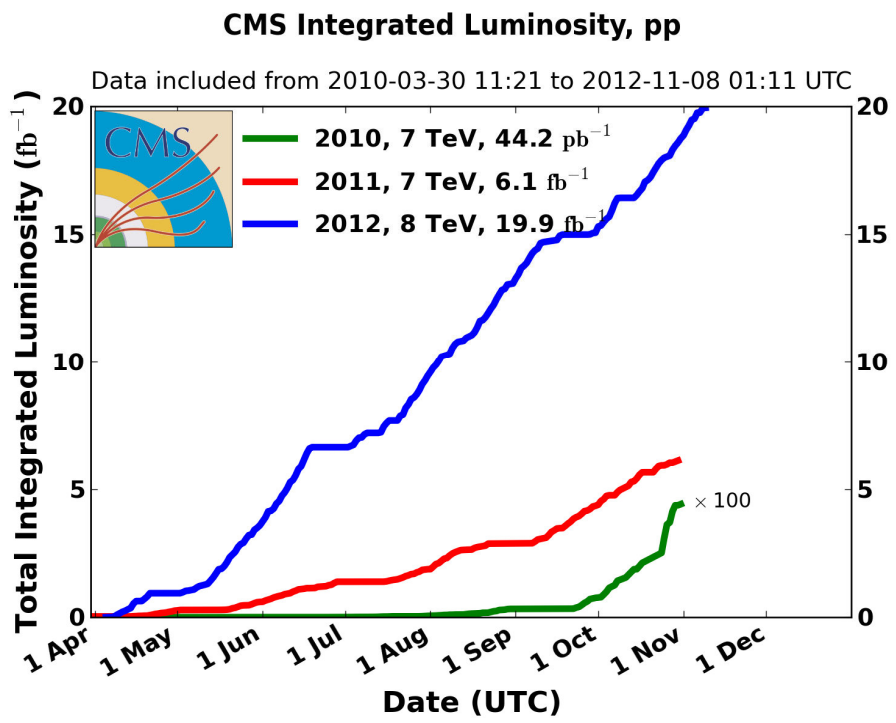

Fig. 5. Integrated luminosity delivered to the CMS experiments in 2010-2012. Prior to the announcement of the discovery of the Higgs boson on July 2012, the data from 2011 was doubled.

A very good vacuum system was required if the beam lifetime was to be largely defined by the $p-p$ cross-section and not scattering off the residual gas in the vacuum pipe. The proton beams are typically stored for about $10 \mathrm{hrs}$. During that time the protons travel around the $26 \mathrm{~km}$ circumference of the LHC ring by about 10 billion $\mathrm{km}$ which is about 65 astronomical units or the distance of a round trip to Pluto. The LHC vacuum is; indeed, better than the vacuum found on the Moon $-10^{-10}$ Torr or only about 3 million molecules $/ \mathrm{cm}^{3}$.

The performance of the LHC is summarized in Fig. 5 which shows the integrated luminosity delivered to CMS as a function of calendar time. The LHC began operation at $7 \mathrm{TeV}$ CM energy on 30 March 2010. In 2010, the LHC accelerator was commissioned and it delivered about $30 \mathrm{pb}^{-1}$ of luminosity to the experimenters. Rapid progress followed in 2011 with the delivery of $6 \mathrm{fb}^{-1}$ followed by the operation of 2012 which delivered about twice the collision rate as in $2011,6.7 \mathrm{fb}^{-1}$ up to the time of the announcement of the Higgs discovery by the ATLAS and CMS experiments. It was this spectacular performance by the LHC which enabled the discovery of the Higgs boson.

\section{The CMS Detector and the Higgs}

Mounting a decisive search for the Higgs boson required both a new and powerful accelerator, the LHC and new and much more complex detectors, ATLAS and CMS. The CMS detector was conceived by a group in $1990^{19}$ and in 1992 the protocollaboration submitted a Letter of Intent (LoI) to construct CMS. ${ }^{20}$ In 1994 the CMS Technical Proposal ${ }^{21}$ was approved and construction could begin upon formal 
approval in 1997. CMS was then constructed by a large and powerful worldwide collaboration and installed $100 \mathrm{~m}$ underground from October 2006 until January 2008.

The CMS detector was explicitly designed to discover the SM Higgs boson if it were to exist. The Letter of Intent states that "A SM Higgs boson with mass between $95 \mathrm{GeV}$ and $150 \mathrm{GeV}$ would be discovered (signal significance $>5 \sigma$ ) via its two photon decay after an integrated luminosity of about $31 \mathrm{fb}^{-1}$. The same integrated luminosity gives a discovery range covering masses from $135 \mathrm{GeV}$ to $525 \mathrm{GeV}$ in the four-lepton $\left(e\right.$ or $\mu$ ) channel.... An integrated luminosity of $100 \mathrm{fb}^{-1}$ (taken at $10^{34} \mathrm{~cm}^{-2} \mathrm{~s}^{-1}$ ) would allow discovery via these channel over the full range between $85 \mathrm{GeV}$ and $700 \mathrm{GeV}$... using decay modes with larger branching ratios $(H \rightarrow W W \rightarrow l v j j)$ and $(H \rightarrow Z Z \rightarrow l l j j)$, should allow the discovery range for an SM Higgs boson to be extended up to $1 \mathrm{TeV}$ for the same integrated luminosity."

The specific choices of detector subsystems also flowed from the requirements for making a decisive Higgs search. The design documents state that: "The choice of a high resolution, high granularity crystal calorimeter for CMS optimizes the chances of discovery in the $m_{H} 80 \mathrm{GeV}$ to $140 \mathrm{GeV}$ range where the $H \rightarrow \gamma \gamma$ decay mode provides the best signature. For the mass range $130<m_{H}<600 \mathrm{GeV}$, the most appropriate search channel is $H \rightarrow Z Z^{*}, Z Z^{*} \rightarrow 2 l^{+} 2 l^{-}$. This entire mass range can be covered with a good safety margin, thanks to the very good muon momentum resolution in the $4 T$ field, the excellent electron energy resolution and the adequate electron and muon geometric acceptance."

Note that, because the $H$ mass is not known and is a free parameter in the SM a general purpose detector was designed. ${ }^{22,23}$ There was guidance from both experimental data and theory. A lower limit of $114 \mathrm{GeV}$ was established at the LEP electron-positron collider at CERN. An upper limit of roughly one TeV was set by general considerations of unitarity in $W+W$ scattering. Within that range of masses the cross-section for production can be accurately predicted because the coupling of the SM Higgs boson to the $W, Z$ and the quarks is fully defined using Eqs. (6) and (7). The predicted Higgs decay widths, Eq. (10), for decays into $W$ and quarks are

$$
\begin{aligned}
\frac{\Gamma\left(\phi_{H} \rightarrow W W\right)}{M_{H}} & =\left(\frac{\alpha_{W}}{16}\right)\left(\frac{M_{H}}{M_{W}}\right)^{2} \beta, \\
\frac{\Gamma\left(\phi_{H} \rightarrow q \bar{q}\right)}{M_{H}} & =\left(\frac{3 \alpha_{W}}{8}\right)\left(\frac{m_{q}}{M_{W}}\right)^{2} \beta^{3} .
\end{aligned}
$$

The Higgs width divided by its mass goes as the square of the Higgs mass for $W$ decays and of the quark mass for decays into quarks. In Eq. (10), $\beta$ is the CM velocity of the decaying particles with respect to $c$ and the power-law dependence on $\beta$ follows from the assumption that the Higgs is a scalar boson. A numerical comparison using Eq. (10) shows that the favored final states are $W W, Z Z$ and top pairs if they are kinematically allowed, or $b$ quark pairs if they are not. Light Higgs decays into $\tau$ leptons are the most favorable to check coupling to leptons. 
Direct Higgs coupling to gluons and photons does not exist because these particles are massless. However, the Higgs can, at higher order, virtually decay into top pairs which then radiate gluons or photons before recombining. These virtual processes lead to a Higgs fractional width to gluons or photons which scales as the square of the Higgs mass, Eq. (11), but with additional factors for strong, $\alpha_{s}$, and electromagnetic, $\alpha$, coupling constants and loop integrals $I$ of order one

$$
\begin{gathered}
\Gamma(H \rightarrow g g) \sim\left[\left(\frac{\alpha_{W}}{8}\right)\left(\frac{M_{H}}{M_{W}}\right)^{2}\right]\left[\left(\frac{\alpha_{s}}{\pi}\right)^{2} \frac{\left|I_{g}\right|^{2}}{9}\right] M_{H}, \\
\Gamma(H \rightarrow \gamma \gamma) \sim\left[\left(\frac{\alpha_{W}}{9}\right)\left(\frac{M_{H}}{M_{W}}\right)^{2}\right]\left[\left(\frac{\alpha}{\pi}\right)^{2} \frac{\left|I_{\gamma}\right|^{2}}{9}\right] M_{H} .
\end{gathered}
$$

All the couplings are well defined in the SM and the resulting total Higgs decay width as a function of Higgs mass ${ }^{24}$ is shown in Fig. 6. Below the $W W$ threshold, the Higgs width is a few $\mathrm{MeV}$. That means the measured width of such a boson would be entirely determined by the experimental resolution for the Higgs mass which in turn would be defined by the energy resolution of the decay products of the Higgs. On the other hand, near $1 \mathrm{TeV}$ Higgs mass the intrinsic decay width is comparable to the mass itself, which relates to the maximum Higgs mass that preserves perturbative unitarity in $W+W$ scattering. In that case the experimental mass resolution is not a critical item.

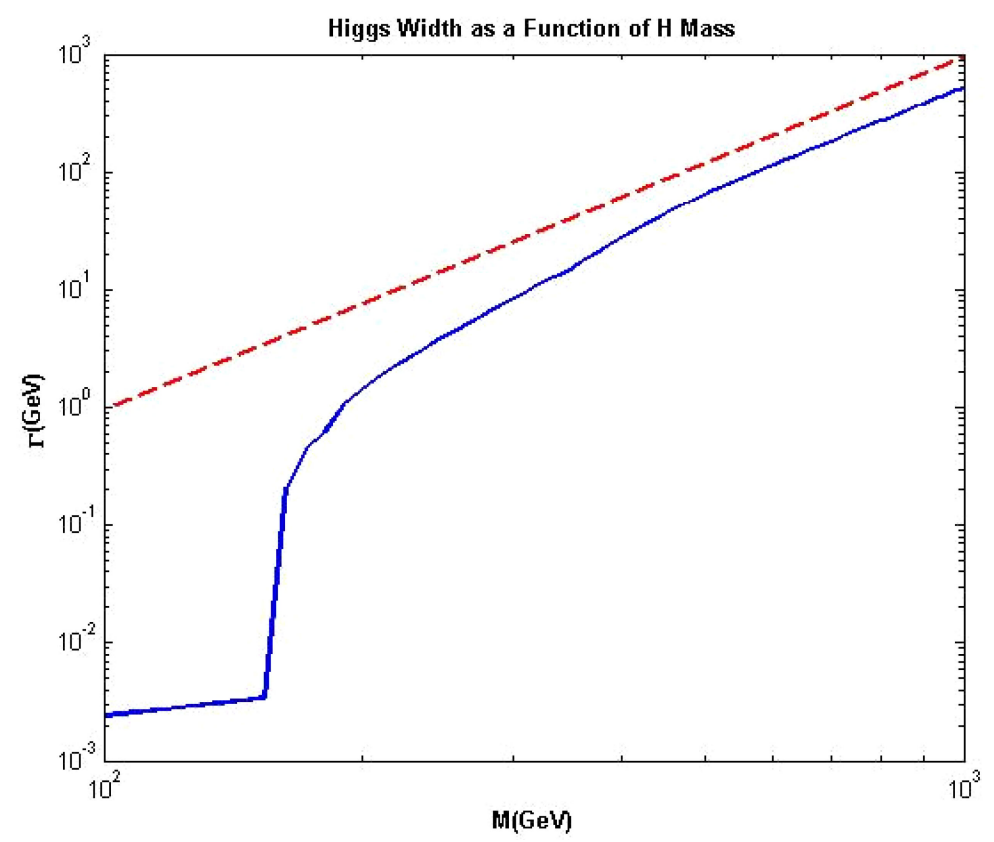

Fig. 6. Higgs total decay width at tree level leading order as a function of the Higgs boson mass. The line indicates a $M_{H}^{3}$ scaling. 


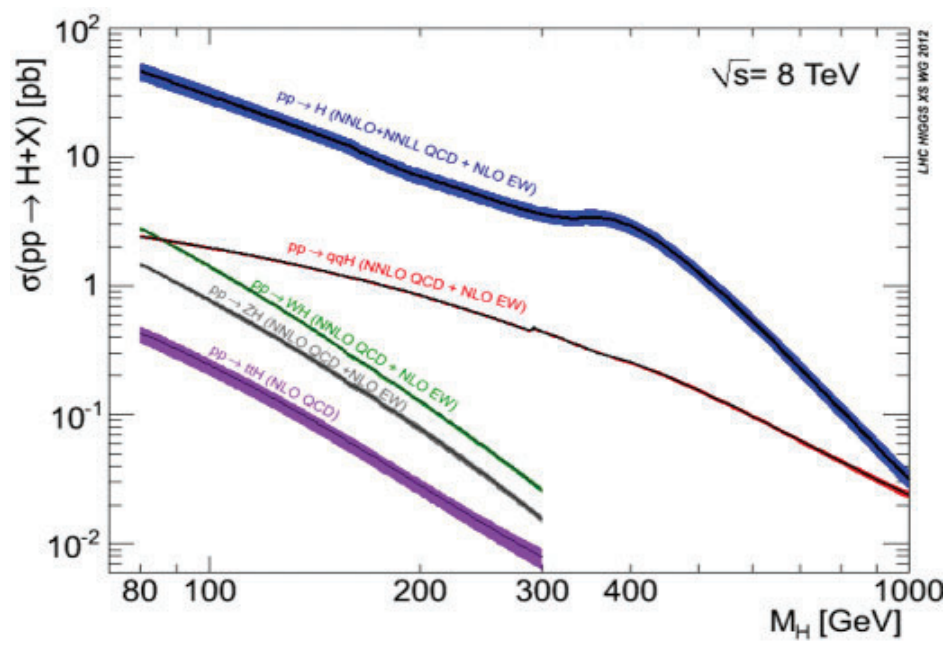

Fig. 7. The predicted Higgs cross-section at $8 \mathrm{TeV}$ CM energy for several distinct production mechanisms, where ggF dominates at low Higgs masses.

The prediction for the Higgs production cross-section follows from the couplings discussed above and from the distribution of partons, gluons and quarks, within the proton. The known Parton Distribution Functions (PDF) were compiled and used in the predictions, ${ }^{25,26}$ which lead to the cross-section predictions. ${ }^{27}$ These crosssections are shown for several production processes in Fig. 7. The largest crosssection arises from gluons in the protons fusing into top pairs which then emit a Higgs boson. This process is called gluon-gluon fusion (ggF). The cross-section at $8 \mathrm{TeV} \mathrm{CM}$ energy is about $20 \mathrm{pb}$ for an $H$ mass of $125 \mathrm{GeV}$. Since the $p-p$ cross-section is about $100 \mathrm{mb}$, in $10 \mathrm{fb}^{-1}$ one needs to consider 1000 trillion interactions and in that sample about 200,000 Higgs bosons will have been produced. The branching ratios are shown in Fig. 8. At $125 \mathrm{GeV}$ mass the largest is for $b$ quark pairs. However, that is not the first choice experimentally since $b$ pairs have an enormous background cross-section from strong interaction production and the mass resolution for the $b$ quark jets is not terribly good, leading to a fairly broad reconstructed Higgs mass distribution.

The $W W$ final state has the next largest branching ratio but since the experimentally viable $l+v+l^{\prime}+v$ final state has missing neutrinos, the mass resolution is not good either. Gluon pairs have too big a strong interaction background and tau leptons are very hard to trigger on and then reconstruct accurately. As noted in the CMS LoI, the $Z Z$ and $\gamma \gamma$ final states offer the best mass resolution. Since the natural width for an $H$ of $125 \mathrm{GeV}$ is of order $\mathrm{MeV}$, this puts a premium on the experimental resolutions.

Since a large signal to background ratio is crucial for discovery, CMS put an focus on precise photon and lepton ( $e$ and $\mu$ decays of the $Z$ ) detection. Using the branching fractions shown in Fig. 7 and the subsequent branching ratio of 


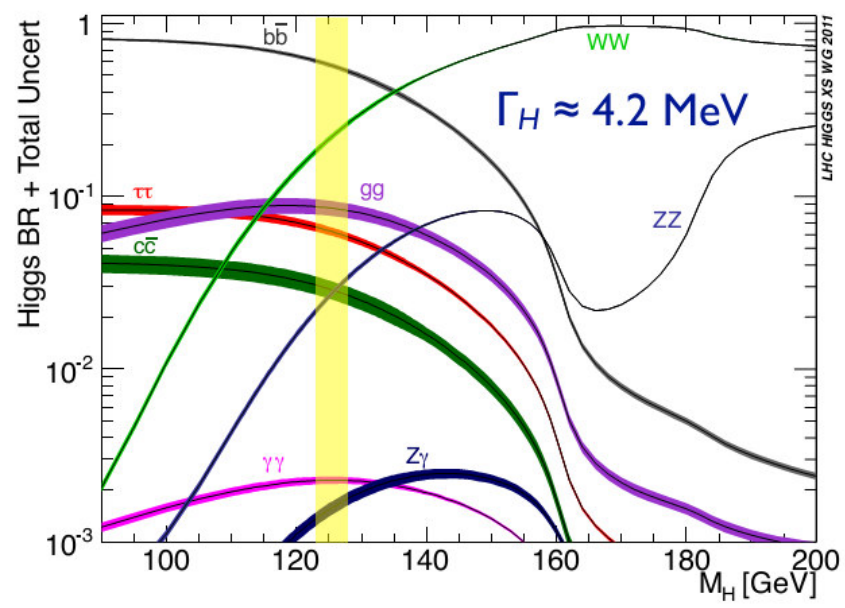

Fig. 8. The branching ratios for an SM Higgs decaying into vector bosons, quarks and leptons.

$Z$ to leptons of $3.36 \%$, of the $200,000 H$ of $125 \mathrm{GeV}$ produced, there are 600 in the two photon final state and 27 in the $Z Z \rightarrow 4 l$ final state. Clearly, the high luminosity of the LHC is required in order to discover the $125 \mathrm{GeV} H$ in relatively clean, narrow mass and background free final states. Since a $1 \mathrm{TeV}$ Higgs has a production cross-section almost 1000 times smaller than a $125 \mathrm{GeV}$ Higgs, that illustrates how difficult it was to design a search over the entire mass range.

\section{The CMS Detector and Analysis - Overview}

The CMS detector is arguably one of the largest and most complex scientific instruments ever built. Nevertheless, it is just sufficient to make a definitive search for the Higgs boson, as seen above by the small number of events expected in a data sample derived from $10 \mathrm{fb}^{-1}$ of integrated luminosity. In describing CMS it is useful to factorize the detector into distinct subsystems, each of which has a role in detecting and measuring the properties of a fundamental particle of the SM. A schematic is shown in Fig. 9 which correlates the SM particles and the detector subsystems that measure some of their properties.

The detector, moving outwards from the interaction point, first has a charged particle tracking system which measures trajectories in a magnetic field, thus determining charge and vector position and momentum. Photons and neutrinos do not register in the tracking, while electrons, muons and jets do. The tracking system is followed by calorimetry which initiates a cascade of electromagnetic (ECAL) or hadronic (HCAL) interactions which absorb almost all the particle energy and thus measure it in a localized angular region of the calorimeters. Electrons and photons register in ECAL, while jets largely register in HCAL. Muons remain after all other particles are absorbed in the calorimetry and are measured in the muon system, in which CMS is embedded in the solenoid magnet return yoke. Finally, neutrinos do 


\begin{tabular}{|c|l|l|l|l|}
\hline $\begin{array}{c}\text { Particle } \\
\text { type }\end{array}$ & Tracking & ECAL & HCAL & Muon \\
\hline$\gamma$ & & & & \\
\hline $\mathrm{e}$ & & & & \\
\hline$\mu$ & & & & \\
\hline Jet & & & & \\
\hline $\begin{array}{c}\text { Et } \\
\text { miss }\end{array}$ & & & $<$ & \\
\hline
\end{tabular}

Fig. 9. Particles of the SM and the detector subsystems that are used to detect and measure them.

not interact at all and their presence must be inferred from a momentum imbalance between the initial and final state, leaving a missing transverse momentum (MET), to be ascribed to that carried away by the neutrinos. Calorimetric coverage goes to pseudorapidity of $|\eta|<5$ (all angles $>0.77^{\circ}$ to the $p$ beams) to insure "hermetic" or almost complete solid angle coverage of the outgoing particles. This coverage ensures that the MET is accurately measured.

The CMS detector is shown in more detail in Fig. 10. The full detector weighs 14,000 tonnes. It consists of silicon tracking and crystal ECAL followed by steel HCAL - all inside the CMS solenoid. The muon system resides in the steel magnetic flux return yoke.

The CMS superconducting solenoid has a central field of $4 \mathrm{~T}$, achieved in 2006 . The stored energy is 3 GJ which is roughly equivalent to $600 \mathrm{~kg}$ of TNT. The solenoid outer radius is $3 \mathrm{~m}$, with a length of $13 \mathrm{~m}$ and inside of which all CMS subsystems are enclosed except the muon system. The superconducting coil is energized by a current of $20 \mathrm{kA}$. The magnet is a large step ahead compared to contemporary solenoids as shown in Fig. 11. A strong magnetic field is essential for accurate charged particle tracking of the electrons and muons from the $Z Z$ decay of the $H$ mentioned above by imparting a large curvature to the particle trajectories.

The pixel silicon detector is closest to the interaction point. The particle rate at a transverse radius of $4 \mathrm{~cm}$ from the beams is about $100 \mathrm{MHz} / \mathrm{cm}^{2}$, scaling roughly as $1 / r^{2}$ to $1 \mathrm{MHz} / \mathrm{cm}^{2}$ at a radius of $50 \mathrm{~cm}$. Clearly, high granularity of the pixels is needed in order to individually recognize the charged particles emerging from the interaction point amidst all the other produced particles. 
D. Green

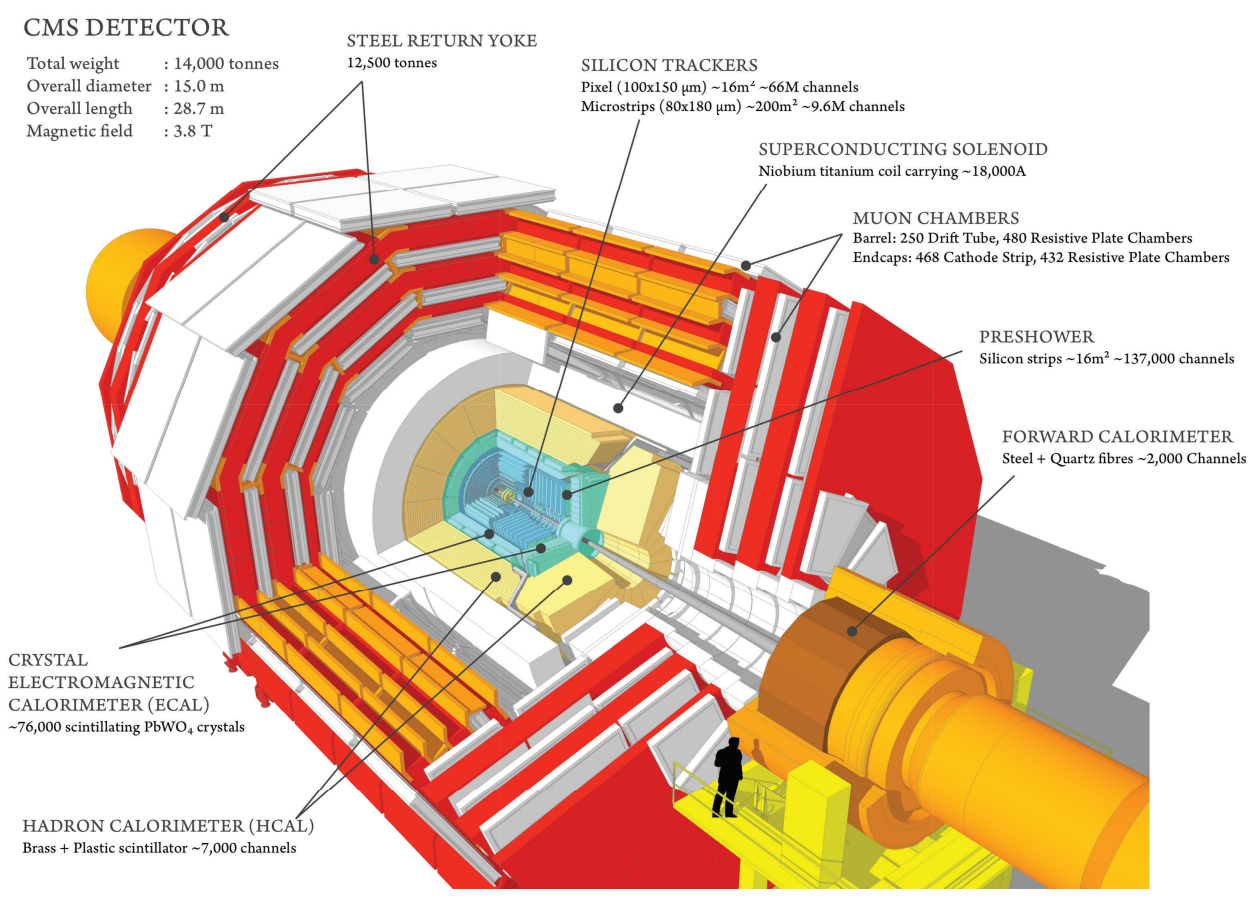

Fig. 10. The subsystems of the CMS detector giving the basic parameters of each.

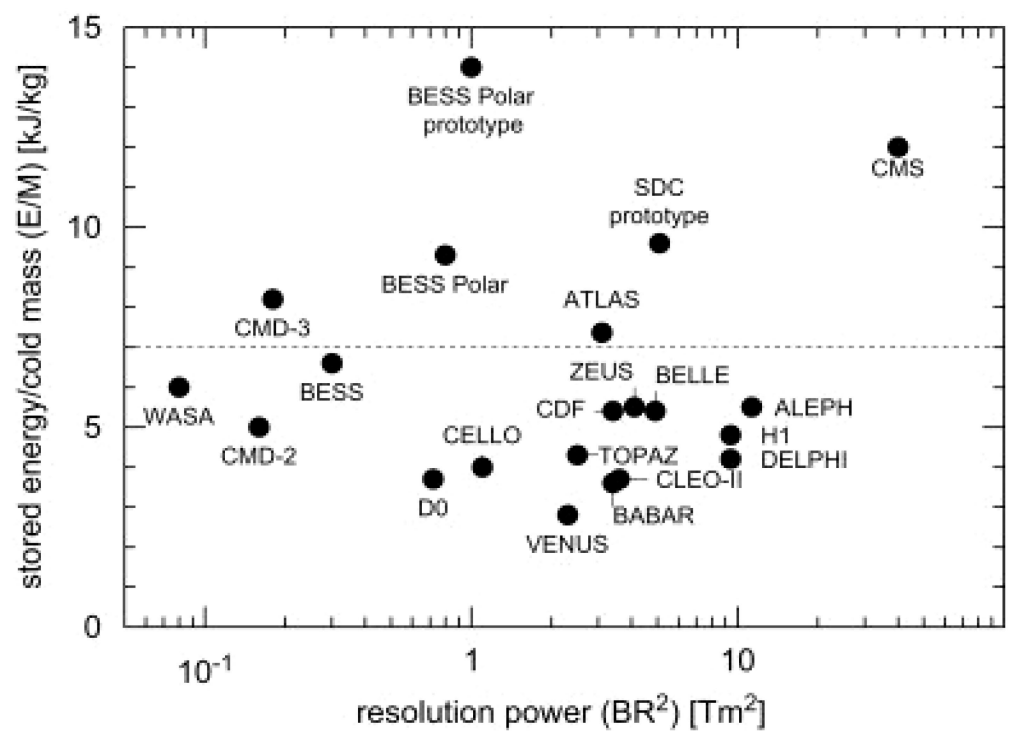

Fig. 11. Stored energy per unit mass as a function of tracking figure of merit, $\mathrm{BR}^{2}$ for several contemporary magnets. 


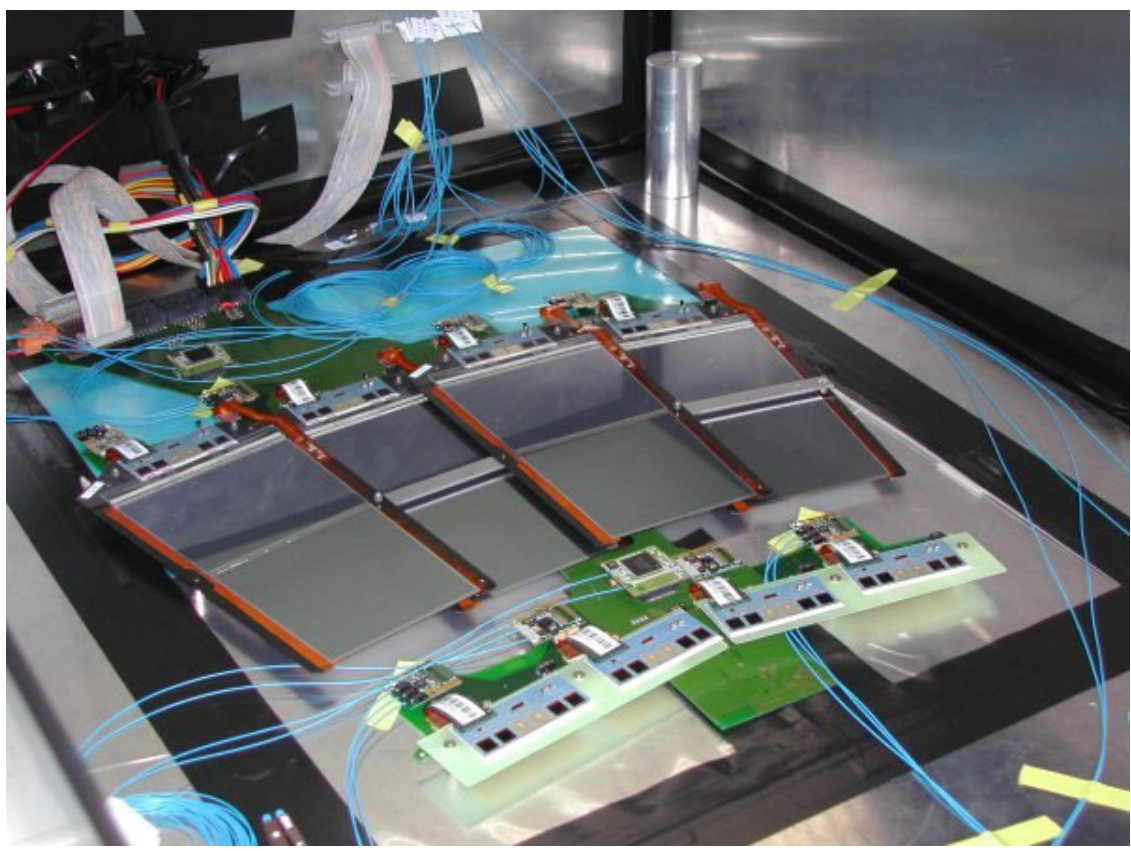

Fig. 12. Test layout of silicon strips intended for the small angle region of CMS. Two wafers are bonded together to yield long strips and thus to reduce the expense of a large channel count.

The silicon pixels are $\sim(100 \mu \mathrm{m})^{2}$ with a granularity limited by cost, power and a tolerable amount of material disturbing the particle trajectories. The probability of a pixel to be hit by a particle in a $25 \mathrm{~ns}$ time window is small even at $4 \mathrm{~cm}$, less than 0.001. At design luminosity the radiation field due to the collisions is quite large. About 1800 charged and neutral particles per 25 ns "bunch crossing" strike the CMS detector. The CMS silicon tracking has a point resolution of about $15 \mu \mathrm{m}$. In CMS there are 66 million pixels and 9.3 million silicon strips giving a detector area of $198 \mathrm{~m}^{2}$ of silicon coverage. This is by far the largest silicon tracking system ever constructed.

The strips are placed at larger radii where the particle density falls off due to its inverse square behavior. The strips are of small size in the coordinate in which the trajectory has curvature, $\phi$, and large in the coordinate which does not, $z$. This layout was adopted to preserve the good momentum resolution but reduce the channel count. A picture of typical strips appears in Fig. 12.

For the ECAL, CMS chose heavy crystals made of $\mathrm{PbWO}_{4}$. The idea was to have the best possible resolution for the photons since they are expected to be a final state of choice for low mass Higgs searches and since the tracking is no help for photons as it is for the determination of electron energy. In all there were 75,848 crystals grown and assembled and all were delivered by March 2008. A picture of the crystals is shown in Fig. 13. 


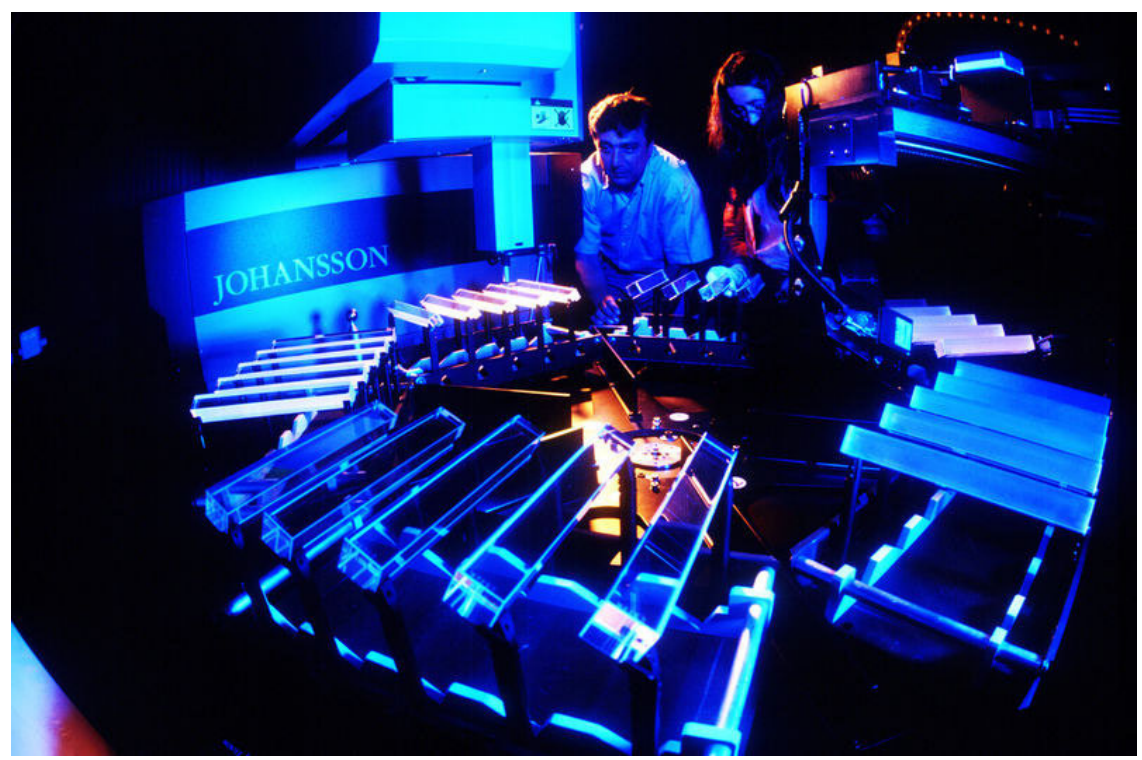

Fig. 13. The ECAL crystals were subjected to rigorous incoming testing, characterization and quality control before being assembled together and installed in CMS.

The other subsystems of CMS were somewhat less innovative. The HCAL was a conventional steel scintillator sampling calorimeter. The issues with it were limited space inside the CMS solenoid for sufficient HCAL depth and radiation hard detectors and transducers, the latter which needed to operate in the 4 T CMS magnetic field. The muon system was chosen to use a conventional gas ionization detector. The very large number of channels, the large surface area to cover and the backgrounds due to muons from collisions and from accelerator effects outside the CMS detector were the issues of this method.

It has been said that in a hadron collider, what is important is "trigger, trigger and trigger." This is especially true at the LHC with its high luminosity. Event selection must be imposed to take the $40 \mathrm{MHz}$ of bunch crossings (1 GHz of interactions) down in two stages to what can be affordably kept on permanent media. On the detector each readout channel (100 million channels) has a pipeline which is $3.2 \mu$ s deep. Fairly coarse "trigger" information is reported to a hardware trigger (L1) which can handle up to $100 \mathrm{kHz}$ of output rate. This output goes to an online processor farm (HLT) which can operate at up to $0.5 \mathrm{kHz}$ of output rate. This is a difficult task, to preserve the physics of interest while being able to retain only one in 20 million interactions. Justice cannot be done here to the work of the CMS trigger experts. ${ }^{28,29}$

The triggered data must be acquired and, given the limited computing resources in any particular region where CMS collaborators reside, distributed throughout the collaboration in order to use all available resources. For a year of data taking, 


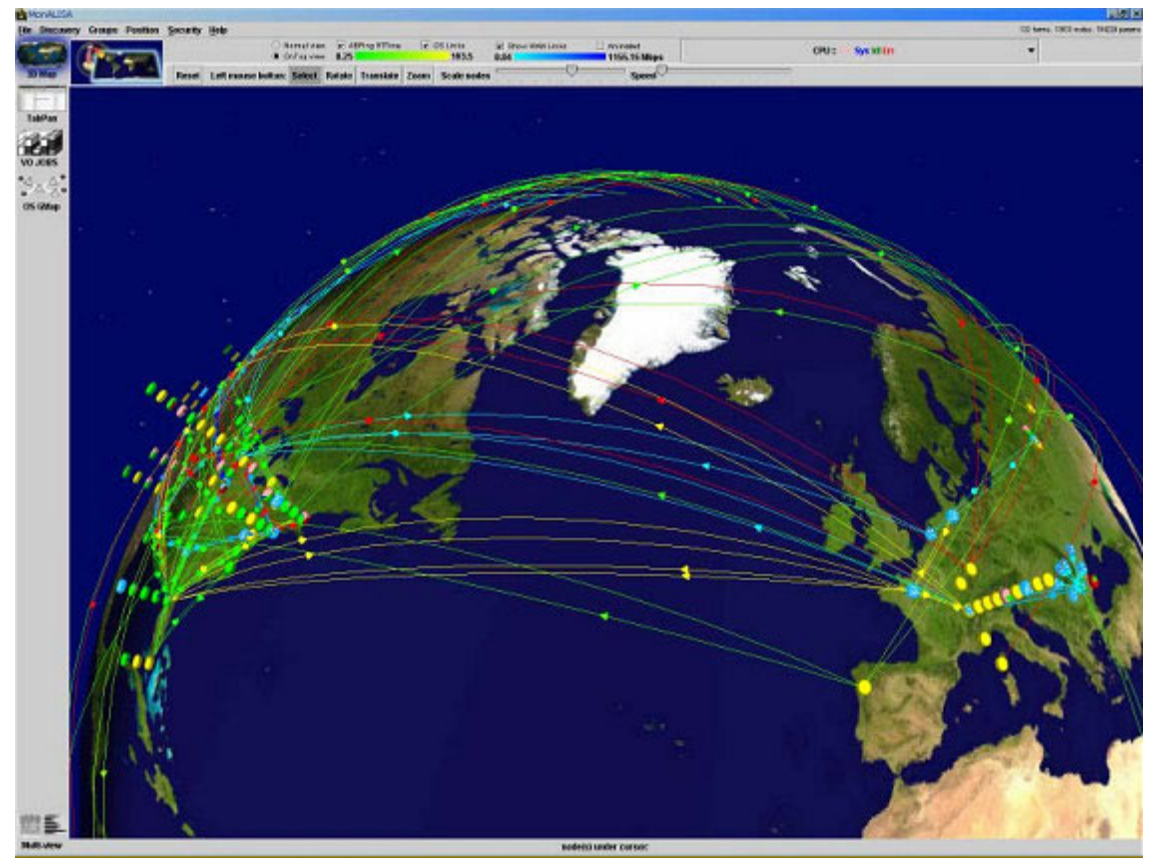

Fig. 14. Schematic showing the data flow for reconstructed events originating in CERN (T0) and going to national or regional sites for further processing (T1).

CMS will write about 10 million GB/year or about 1 million CD/year. In order to enable the entire collaboration to work on the physics CMS and ATLAS planned to distribute reconstructed data worldwide with a very short latency, less than $48 \mathrm{hrs}^{29}$ as a goal. The data have a first pass reconstruction at CERN (Tier 0) and are then distributed to national/regional computing centers (Tier 1) for further processing. Smaller specialized datasets are then sent to local clusters of collaborators within a country (Tier 2). Building up this worldwide grid has been a wonderful enterprise and a great success. ${ }^{30} \mathrm{~A}$ cartoon of the data transfers is shown in Fig. 14. The Worldwide LHC Computing Grid connects 100,000 processors in 34 countries with ultrahigh-speed data transfers.

CMS as collaboration grew to 3000 scientists and engineers from 180 institutions located in 40 countries. This team was responsible for installing, testing and commissioning the entire CMS detector. The data, in turn, were acquired, reconstructed and skimmed and sent to the entire team in almost real time using the computing grid and distributed farms of processors.

\section{Cosmic Ray Data Taking and Other Preparations in CMS}

CMS had documented the detector in a comprehensive technical description of its performance prior to any data taking. ${ }^{31}$ ATLAS and CMS had also described their detector subsystems in detail. ${ }^{32}$ CMS began to take data using the available cosmic 
ray flux in 2008 and 2009 while waiting for full operation of the LHC. The cosmic ray data allowed CMS to achieve a preliminary alignment and calibration of parts of the detector prior to taking beam. Trigger and data acquisition systems could then also be exercised and partially commissioned. More than one billion cosmic ray triggers were taken. ${ }^{33}$ Using cosmic rays as preparation, the CMS detector was very rapidly commissioned to be ready to explore the physics of $7 \mathrm{TeV} p-p$ interactions.

In addition, it is of vital importance to model a complex experiment with a very complete Monte Carlo description of the physics processes and the specific detector elements. The physics processes were taken from Monte Carlo models, PYTHIA, ${ }^{34}$ Herwig $^{35}$ and MADGRAPH ${ }^{36}$ among others. Those processes were then propagated through the CMS detector model using the GEANT ${ }^{37}$ Monte Carlo. The detector model was of unprecedented complexity in an attempt to make as complete a simulation of CMS as possible. Comparing these simulated data to actual data for well known physics processes then gives the experimenters confidence in their understanding of the CMS detector performance. The assembled CMS detector is shown in the photograph of Fig. 15 where a person in the frame sets the size scale of CMS.

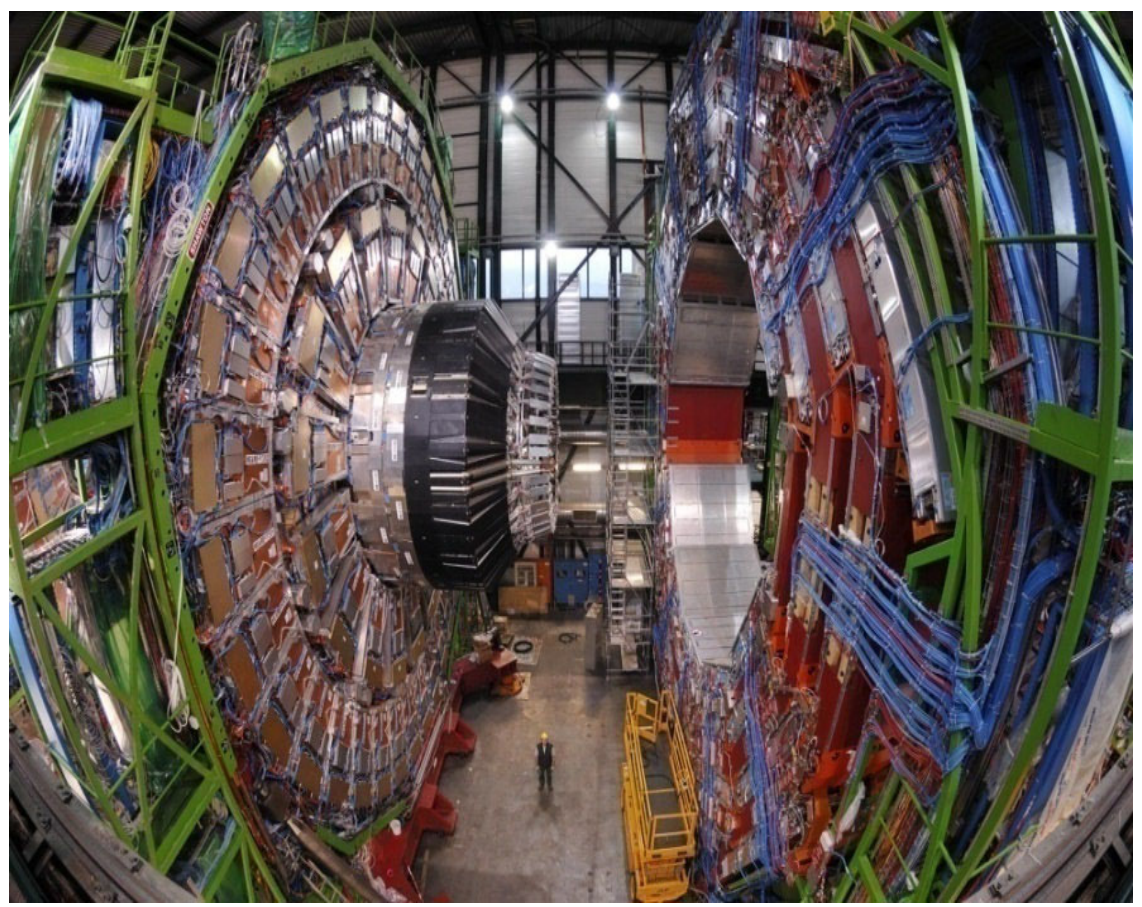

Fig. 15. Photo of CMS upon completion before it was fully assembled by moving the endcaps on the left into the barrel magnet bore seen on the right. 
Having created these tools, and prior to data taking in 2010 the Monte Carlo models were used to push through simulated physics analyses using the models and thus creating a "Physics Technical Design Report" 38 which gave the expected performance of CMS for an ensemble of SM physics processes.

\section{The 2010 Start of $7 \mathrm{TeV}$ Data Taking}

Early data taking at $7 \mathrm{TeV}$ in 2010 allowed CMS to "rediscover the standard model" by commissioning electron algorithms, ${ }^{39,40} \mathrm{MET}$ reconstruction, ${ }^{41}$ jet energy, ${ }^{42}$ jet clustering of jet fragments, ${ }^{43}$ finding decays in $b$ quark jets, ${ }^{44}$ using the full detector in particle flow techniques, ${ }^{45,46}$ confronting pileup of collisions at high luminosities in jets ${ }^{47}$ and defining and debugging tau lepton reconstruction. ${ }^{48}$ Muons had been shaken down using the cosmic ray data in 2009. A few examples of finding the fundamental physics objects are given below.

All the silicon tracking system of CMS provides very complete charged particle tracking. In particular, the system allows CMS to look for the decays of produced heavy quarks such as $b$ quarks. Since the point error is only $15 \mu \mathrm{m}$, while the rest lifetime distance of a $b$ quark is about $470 \mu \mathrm{m}$, efficient tagging of $b$ decays is possible. In Fig. 16, is shown the reconstructed primary (production point) and secondary (decay) vertex. The ellipses indicate the estimated errors on the primary and decay vertices. As expected, the vertex pixel tracker allows CMS to efficiently tag $b$ quark decays.

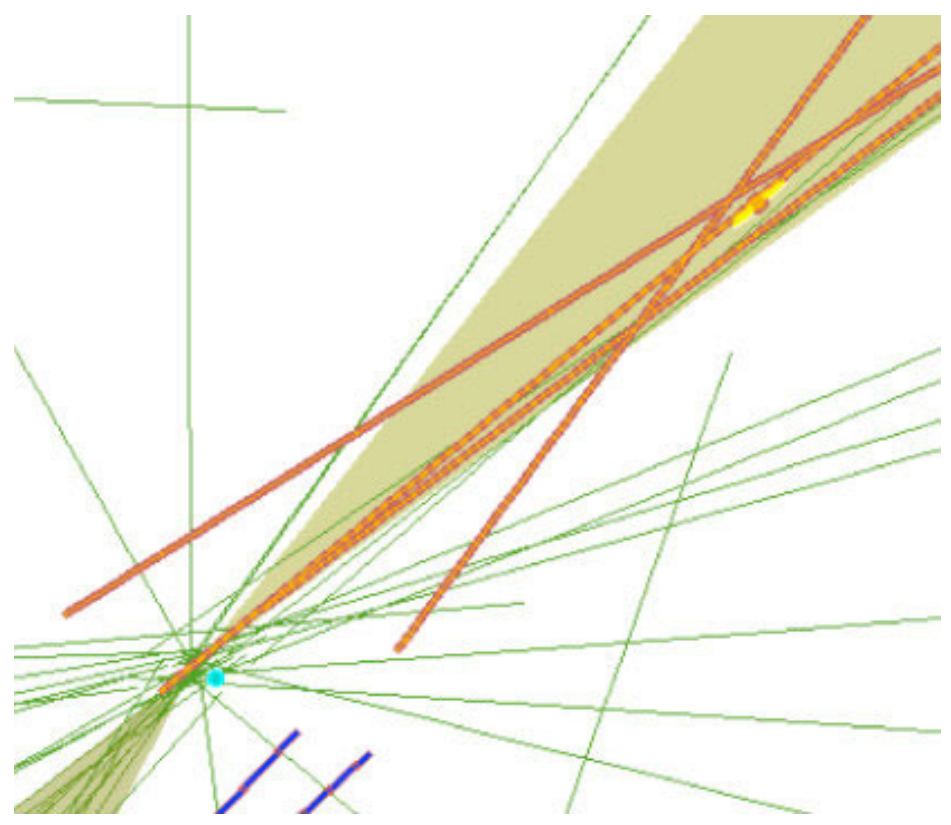

Fig. 16. Reconstructed primary and secondary vertex in CMS with error ellipses displayed. 


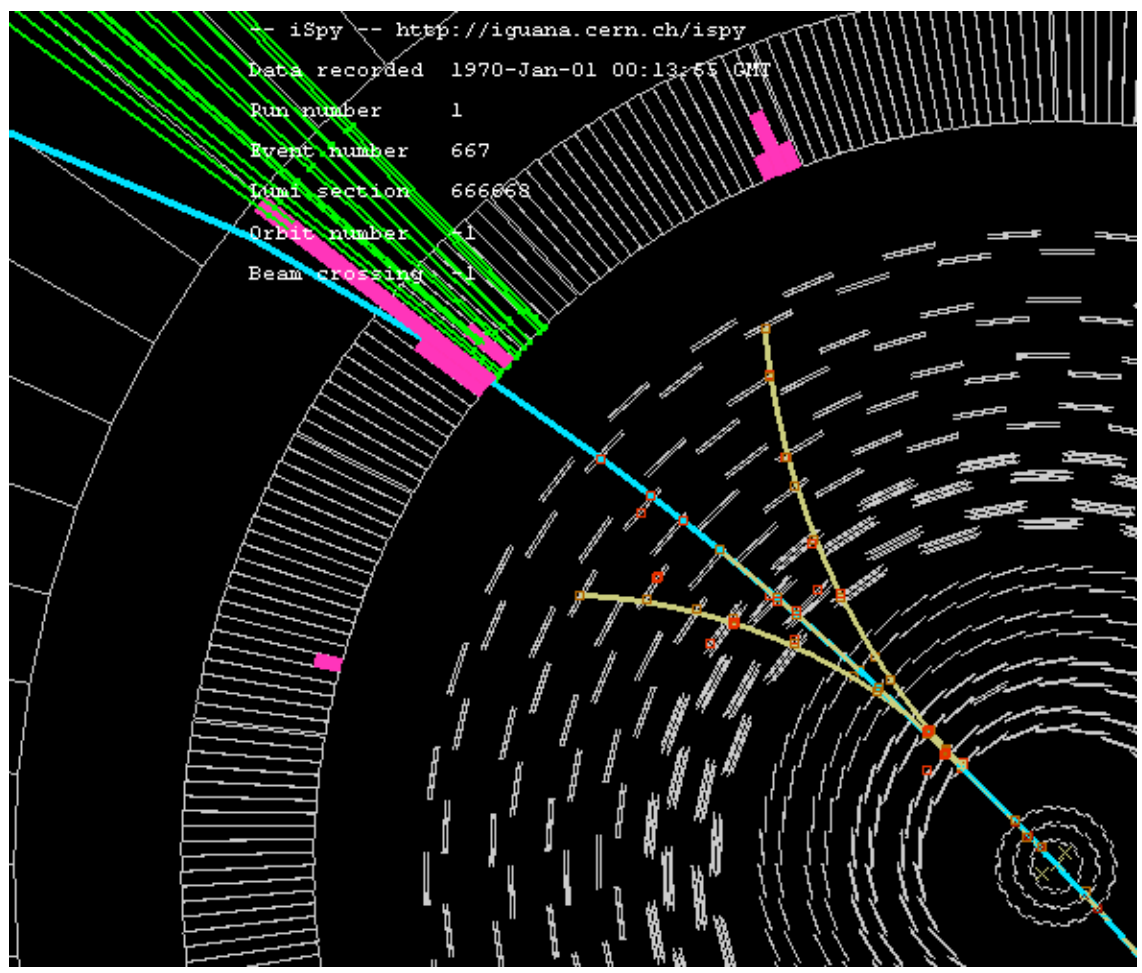

Fig. 17. CMS evens containing an electron which radiates in the tracker material prior to being absorbed in the ECAL.

Electrons and photons appear as ECAL energy deposits with and without associated charged tracks indicating electrons and photons, respectively. Additionally, the electrons may radiate photons which can then convert in the material of the tracking system. This makes the physics object definition more complex, as illustrated in Fig. 17. The electron energy is measured redundantly in the tracker and then the ECAL. That redundancy allows CMS to use the electrons as a proxy for photons and to monitor the reconstruction efficiency and energy resolution for photons.

The MET is a global variable since all energy in the final state must be measured and compared to that in the initial state. Therefore, the MET resolution samples the energy measuring capability of the entire CMS detector. By using particle flow techniques this resolution is minimized in event types that contain no real missing energy (neutrinos). The resulting MET resolution as a function of the total transverse energy in the event is shown in Fig. 18. The resolution is a few percent of the total energy, indicating that CMS has established good measurements of the energy deposited in the entire detector and that an accurate Monte Carlo model exists for this global variable. 


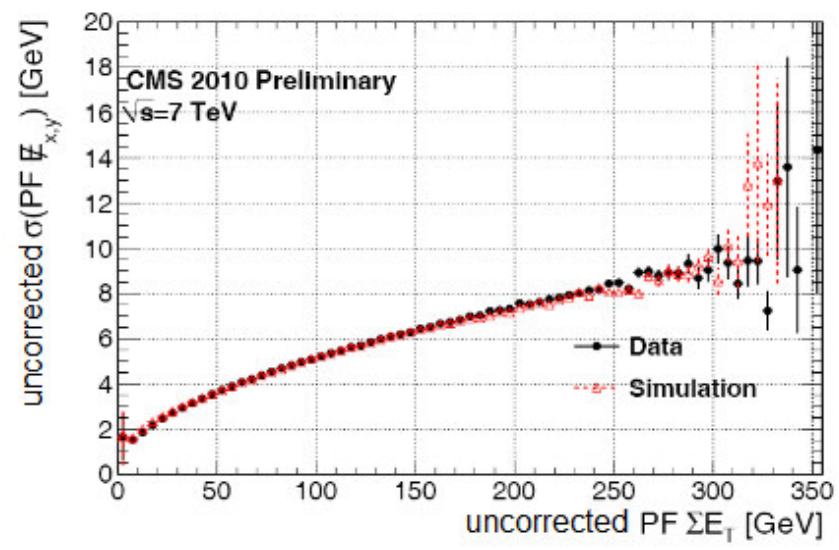

Fig. 18. Resolution of the Gaussian core of the MET distribution in CMS as a function of the total particle flow energy found in the event.

There is a maxim in biology that "ontogeny recapitulates phylogeny," or that the developing fetus retraces the evolutionary path from simpler to more complex forms. In the case of CMS, after the basic physics objects, such as electrons, were commissioned, known reaction cross-sections and kinematic distribution from the SM could be compared to the Monte Carlo models and an ensemble of physics processes and "standard candles" were thus established. An example is shown in Fig. 19, where the dimuon mass spectrum is displayed. Resonant structures are seen and identified as bound states of $u$ and $d$ quarks, $s$ quarks, $c$ quarks, $b$ quarks and finally the electroweak $Z$ boson. In some real sense this early data spans the development of high energy physics from the 1960s up to the mid 1980s which was indicated in Figs. 1 and 2.

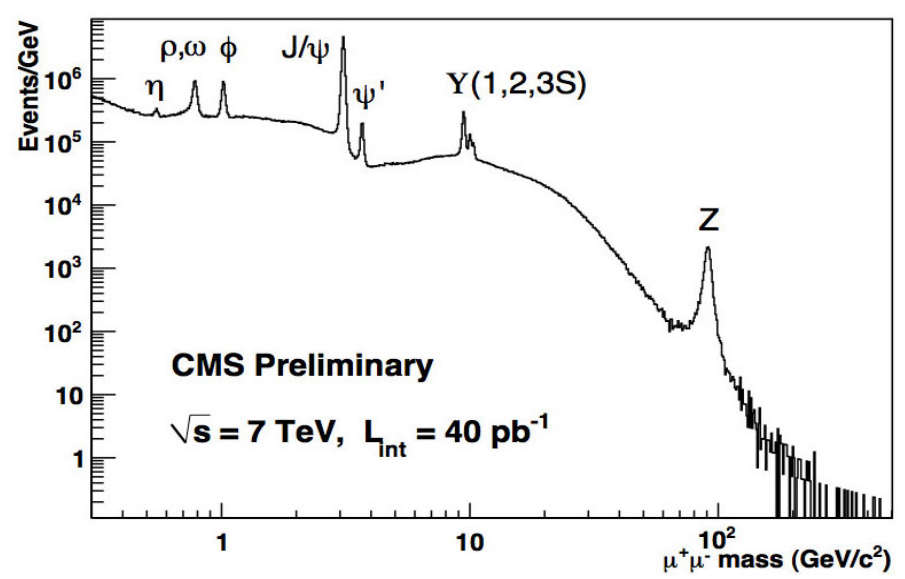

Fig. 19. The dimuon mass spectrum measured in CMS. Several resonant structures, which correspond to bound states of light and heavy quarks are evident. 
The mass and intrinsic widths of the $Z$ resonance are very well known. Therefore, the $Z$ signal can be used to check the momentum scale of the electrons, muons and tau leptons as well as the momentum resolution. In addition, by triggering on a single lepton, CMS was able to measure the trigger and reconstruction efficiencies of the leptons using the second lepton to probe whether it was triggered and reconstructed. These techniques validated the models which CMS had made of the physics processes already known in the context of the SM. The electrons which are $Z$ decay products are also used as proxies for the photons.

For the validation of top quark reconstruction, the top offers an example where many physics objects are needed since the top decays into a $W$ plus a $b$ quark. The $W$ then decays into a lepton plus a neutrino or into a pair of quarks. Thus the top quark-antiquark final state can contain a lepton, missing energy, jets and $b$ quark jets. The cross-section to produce top pairs was well measured in 2010, although the integrated luminosity of $40 \mathrm{pb}^{-1}$ did not allow CMS to explore other rare, low cross-section processes. Finally, the 2010 run allowed CMS to commission the determination of the absolute luminosity, via the method of "van der Meer scans." 49,50

In addition, the LHC experimenters in CMS and ATLAS adopted a common set of multivariate tools $\mathrm{s}^{51}$ and agreed to definitions of confidence limits that were to be used in searches. ${ }^{52,53} \mathrm{~A}$ common set of statistical tools was adopted by the LHC experiments which made cross coordination between ATLAS and CMS much simpler and more transparent. ${ }^{54}$ Thus, most of the tools needed in the Higgs search were in place at the end of the 2010 data taking period.

\section{The 2011 Data Taking at $7 \mathrm{TeV}$ - Higgs Hunt Hints}

The 2011 data taking saw a very large increase in the instantaneous luminosity, which resulted in an integrated luminosity for 2011 in excess of $5 \mathrm{fb}^{-1}$, an increase of a factor about 125 fold. The excellent performance of the LHC allowed CMS to begin to explore rare processes and start the explicit search for the Higgs boson. The state of the art at the Tevatron at that time was known ${ }^{55}$ to exclude masses near $160 \mathrm{GeV}$.

The cross-section for production of a pair of top quarks uses many final state physics objects and CMS measurements are displayed in Fig. 20 for different final state decay products. The precision of these measurements shows that the CMS physics objects are being robustly and accurately measured and give confidence that a comprehensive Higgs search can begin.

The increased luminosity allowed CMS to search for new particle states over a wide variety of possibilities. One such example is shown in Fig. 21, where a sequential $Z^{\prime}$ boson which decays into a pair of top quarks is looked for. ${ }^{56}$ Limits approaching the $1 \mathrm{TeV}$ mass scale were set for this and many other processes.

The increase in sensitivity from 2010 to 2011 analyses is illustrated in Fig. 22 which shows electroweak cross-sections for single vector bosons and for pairs of 


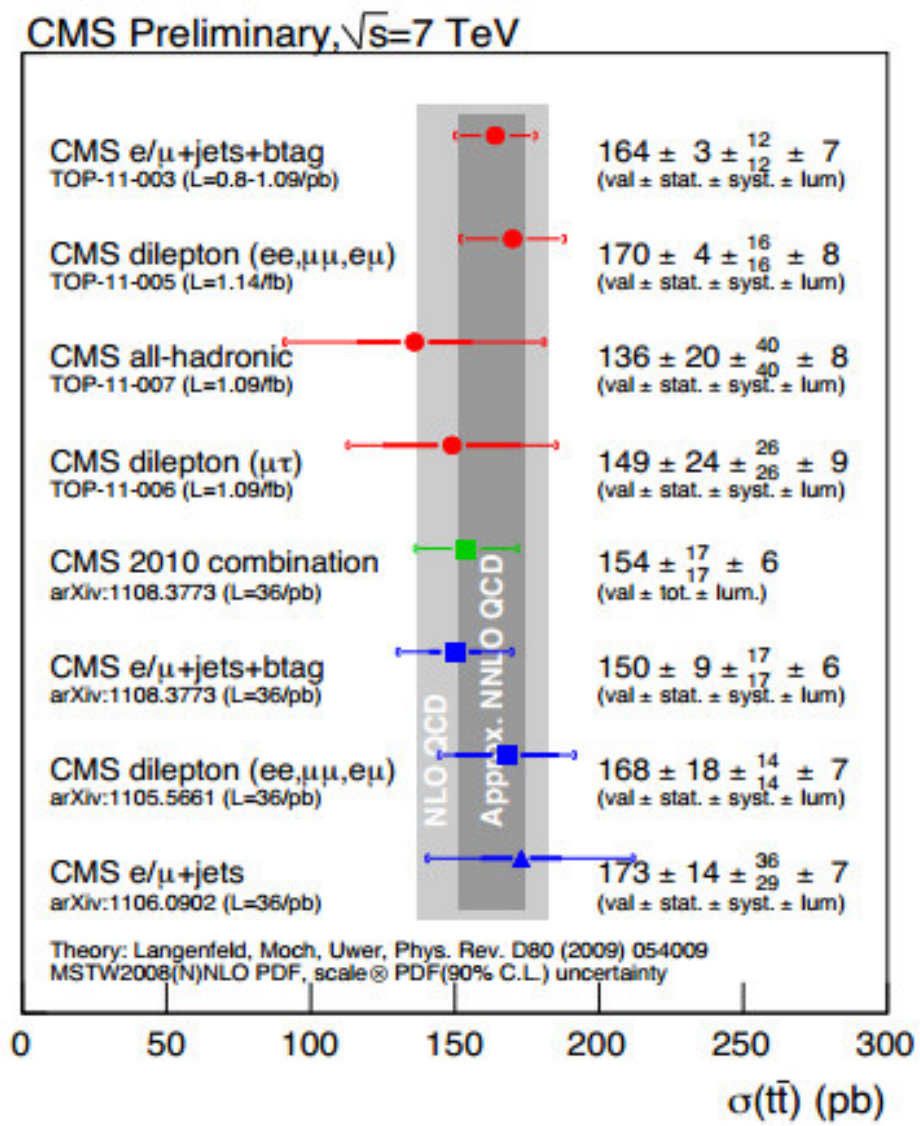

Fig. 20. CMS measurements of the top quark mass using leptons, quark jets and $b$ quark identification. The overall precision on the top mass measurement is comparable to the theoretical uncertainties of the measurements.

bosons. These cross-sections span five orders of magnitude and begin to reach the sensitivity range needed for the discovery of a light Higgs boson. Since vector boson pairs are an irreducible background for Higgs searches in the bi-boson final states, it is important to fully understand and measure these backgrounds.

In fact, CMS observed indications of a low mass Higgs ${ }^{57}$ as did ATLAS. ${ }^{58}$ The combination of both experiments was published ${ }^{59}$ but the results, although tantalizing, were not of sufficient statistical accuracy to claim a discovery. Because the excess of events was seen in 2011 at low mass, CMS could confidently predict that an additional $5 \mathrm{fb}^{-1}$ of data in 2012 would allow a definitive discovery, if the indications of a real particle state were confirmed and were not just due to a statistical fluctuation. For the final states with the best mass resolutions, $Z Z$ and $\gamma \gamma$, a combined "five standard deviation" level of confidence in the result could be expected for a light Higgs as illustrated in the predicted sensitivities shown in Fig. 23. 


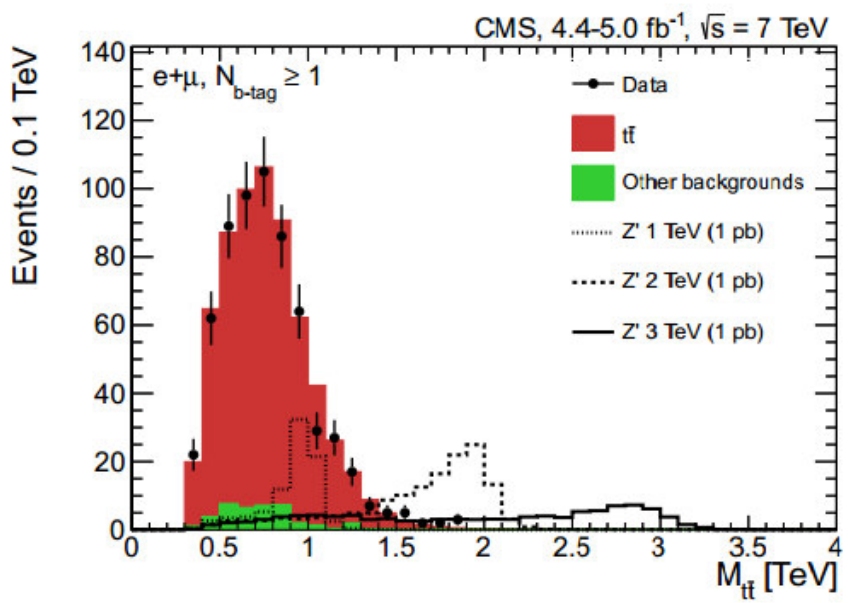

Fig. 21. Mass spectrum of top pairs in CMS and spectra of $Z^{\prime}$ bosons of 1,2 and $3 \mathrm{TeV}$.

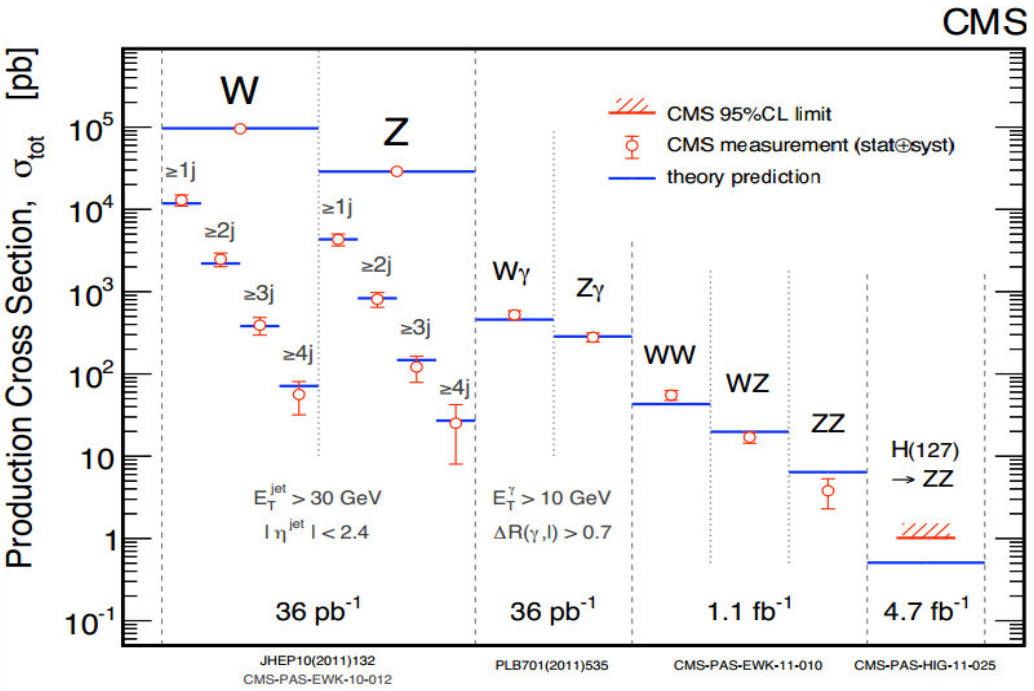

Fig. 22. Cross-sections for electroweak processes measured in CMS in 2010 and 2011.

Because of this extrapolation of the 2011 data taking, CMS "blinded" the analyses by not examining the low mass region near the location of the excess events seen in 2011. In fact, the groups examining the different final states did not communicate with one another during this period in an attempt to enforce statistical independence of the different analyses. Finally, ATLAS and CMS did not communicate any result obtained between the 2011 data analyses and the time of "unblinding," June 2012, as a way to attempt to ensure statistical independence between the two experiments. All these methods were adopted as a way to limit any bias in the experimenters who were examining the new data taken in 2012 . 


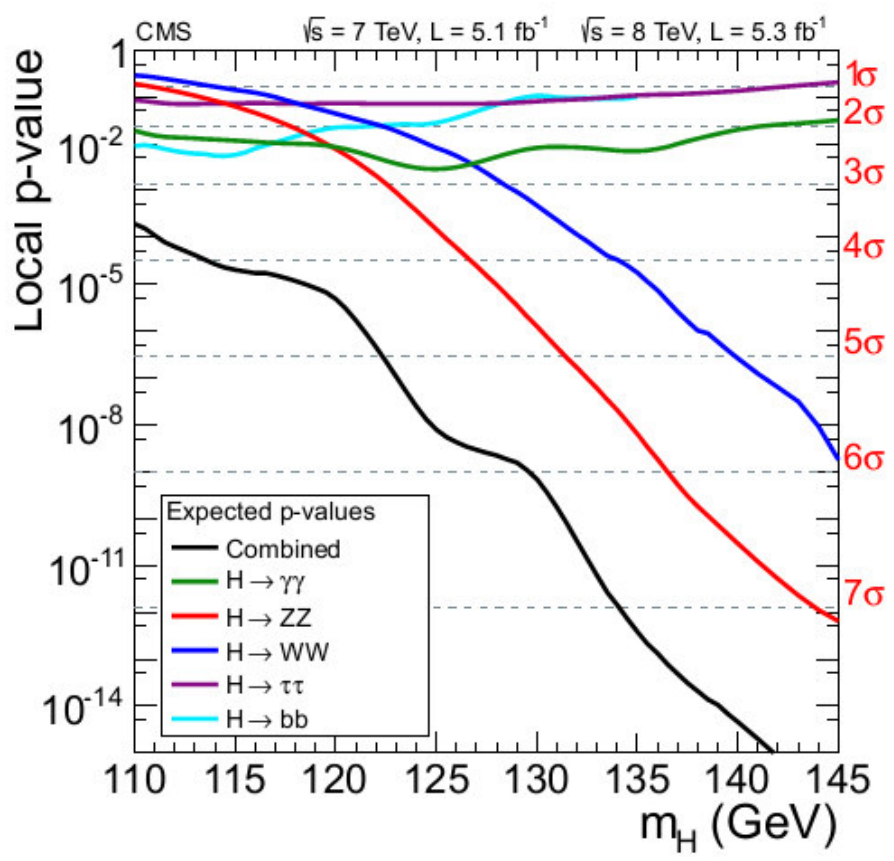

Fig. 23. Expected statistical significance for a Higgs search in different final states with $5 \mathrm{fb}^{-1}$ of additional data taken in 2012 as a function of the mass of a light SM Higgs boson.

\section{Data Taking in 2012 - The Higgs Discovery}

Data taking in 2012 reached an instantaneous luminosity which was twice that attained in 2011. Since a discovery was predicted to become feasible when the 2011 dataset was doubled, that point could already be attained about half way through the 2012 calendar year. Indeed, after the June 2012 "unblinding" both CMS and ATLAS found that the excess of events at low Higgs mass was reinforced and both CMS $^{60}$ and ATLAS ${ }^{61}$ announced the discovery of a new particle on 4 July 2012. The Fermilab Tevatron experiments, CDF and D0 completed their analyses after the Tevatron was turned off at the end of 2011 and announced a combined result in the $b$ quark pair final state at a statistical level below that needed for discovery at the same time. ${ }^{62}$

Recall from the estimates made above that, neglecting detection and reconstruction efficiencies, the expected number of two photon $H$ events is 600 , while the expected number of four-lepton $Z+Z^{*}$ events is 27 after $10 \mathrm{fb}^{-1}$ of data logging for a $125 \mathrm{GeV}$ SM $H$ mass. These are the final states with a good $H$ mass resolution and which are thus expected to give the most sensitive search results. A candidate two photon event is displayed in Fig. 24. The photons are isolated from the accompanying charged particle tracks (orange trajectories) and have little HCAL energy, shown as blue bars, in the photon directions which are indicated by the energy deposits in the ECAL shown as green bars. 


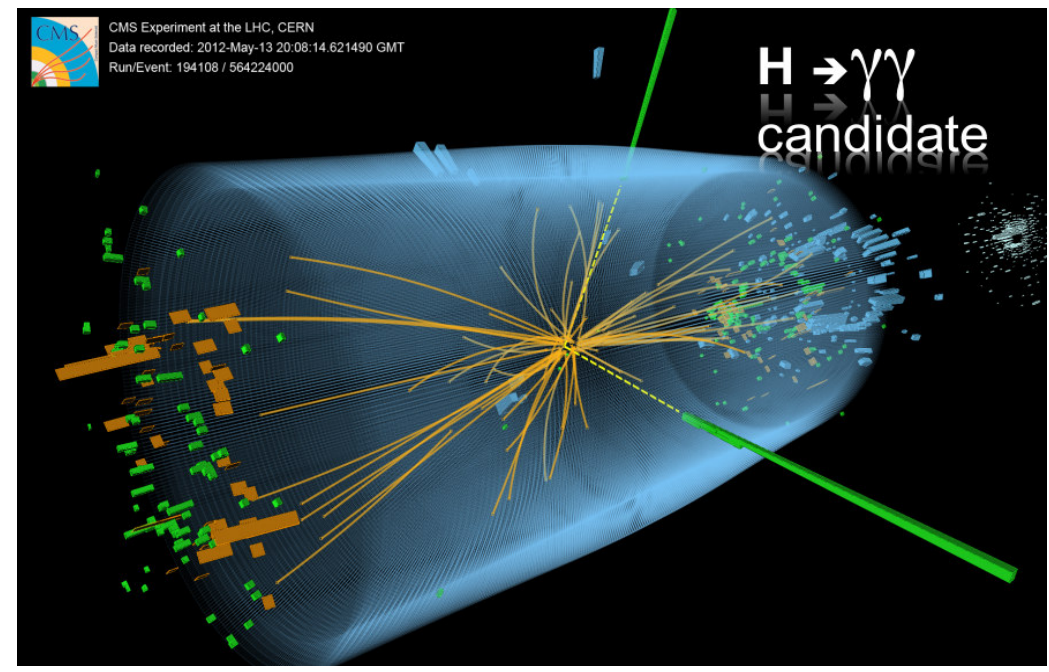

Fig. 24. (Color online) Candidate $H$ decays into two photons. The ECAL energy is shown in green where the height of the bar is proportional to the photon energy.

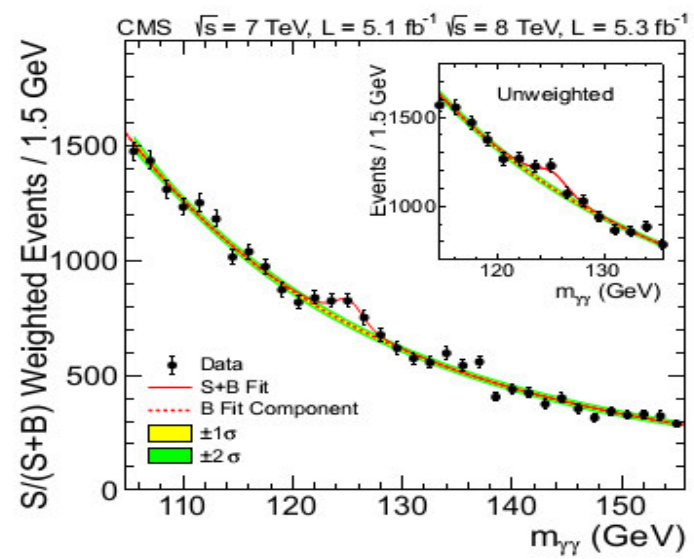

Fig. 25. Mass spectrum of two photons seen in CMS. Events are weighted according to their expected mass resolution. A systematic error band is also indicated.

The invariant mass spectrum of these two photon candidates is shown in Fig. 25. The full dataset for 2011 and the additional 2012 data are used. The curve is the combined shape for a smoothly falling background and a resonant shape at a mass of about $125 \mathrm{GeV}$ plotted assuming that the intrinsic width of the state is small, about $4 \mathrm{MeV}$, as predicted for an SM Higgs boson. The yield for the resonant state is comparable to the 600 events times trigger and reconstruction/selection efficiency that is expected for an SM Higgs boson. The two photon data establishes that the state is narrow, has a mass about $125 \mathrm{GeV}$ and a production cross-section times decay branching ratio consistent with that expected for an SM Higgs boson. 


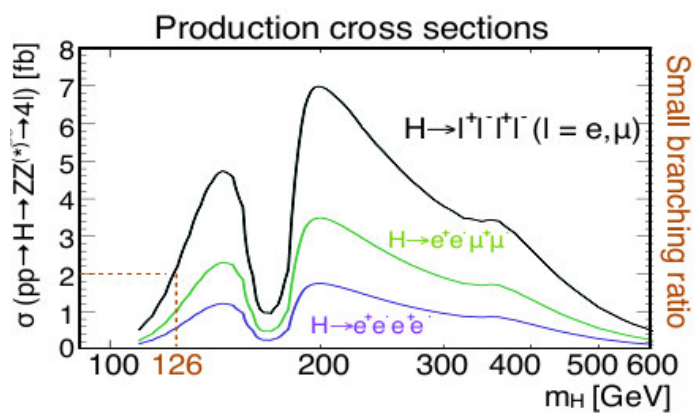

Fig. 26. Cross-section times branching ratio for the four-lepton final state in $H$ production and decay both on shell and off shell for the $Z$ bosons.

The other most sensitive final state, the decay of an $H$ into $Z+Z^{*}$ with subsequent di-lepton decays of both $Z$ was simultaneously explored. With a central mass of $91 \mathrm{GeV}$, on shell $Z+Z$ decays are not possible and at least one $Z$ must be off shell, falling in the low mass tail of the Breit-Wigner resonant $Z$ line shape. The predicted cross-section is shown in Fig. 26 for both on shell and off shell decays. The largest rate for an $H$ signal is just above the threshold for on shell $Z+Z$ decays, while the off shell decays are somewhat suppressed. At a $125 \mathrm{GeV}$ mass, the $2 \mathrm{fb}$ cross-section means that only about 20 events are expected even neglecting the acceptance, reconstruction and selection efficiencies. On the other hand, the mass resolution is very good, making this decay mode very important.

A candidate four-lepton event is displayed in Fig. 27. Because of the resonant mass shape of the $Z$ and because the $\mathrm{SM} Z+Z$ rate is quite small, this final state has a much larger signal to background ratio than the two photon final state. That gain

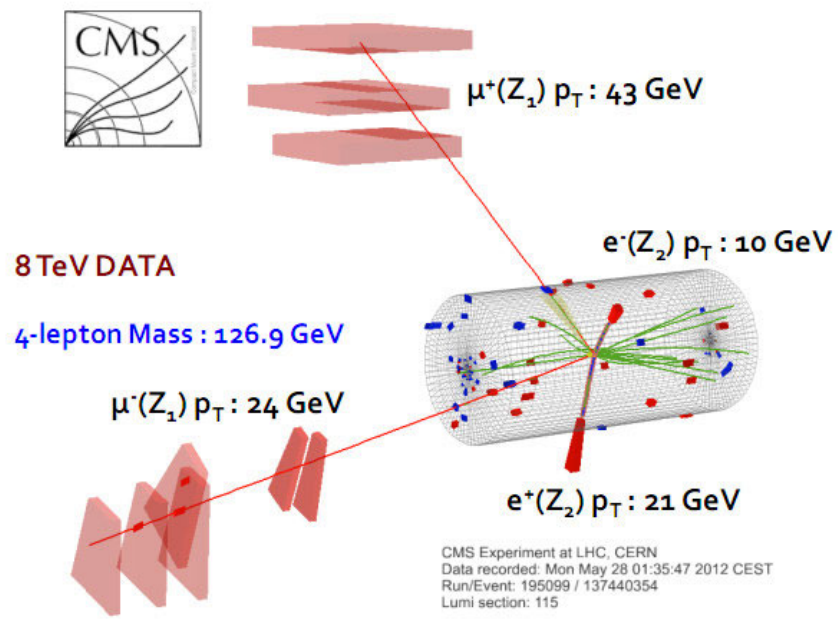

Fig. 27. Candidate $Z+Z^{*}$ event where one $Z$ decays into muons and the other, the $Z^{*}$, decays into electrons. 
D. Green

\section{$2 \mathrm{D}$ analysis using $\left\{\mathrm{m}_{41,}, \mathrm{MELA}\right\}$}
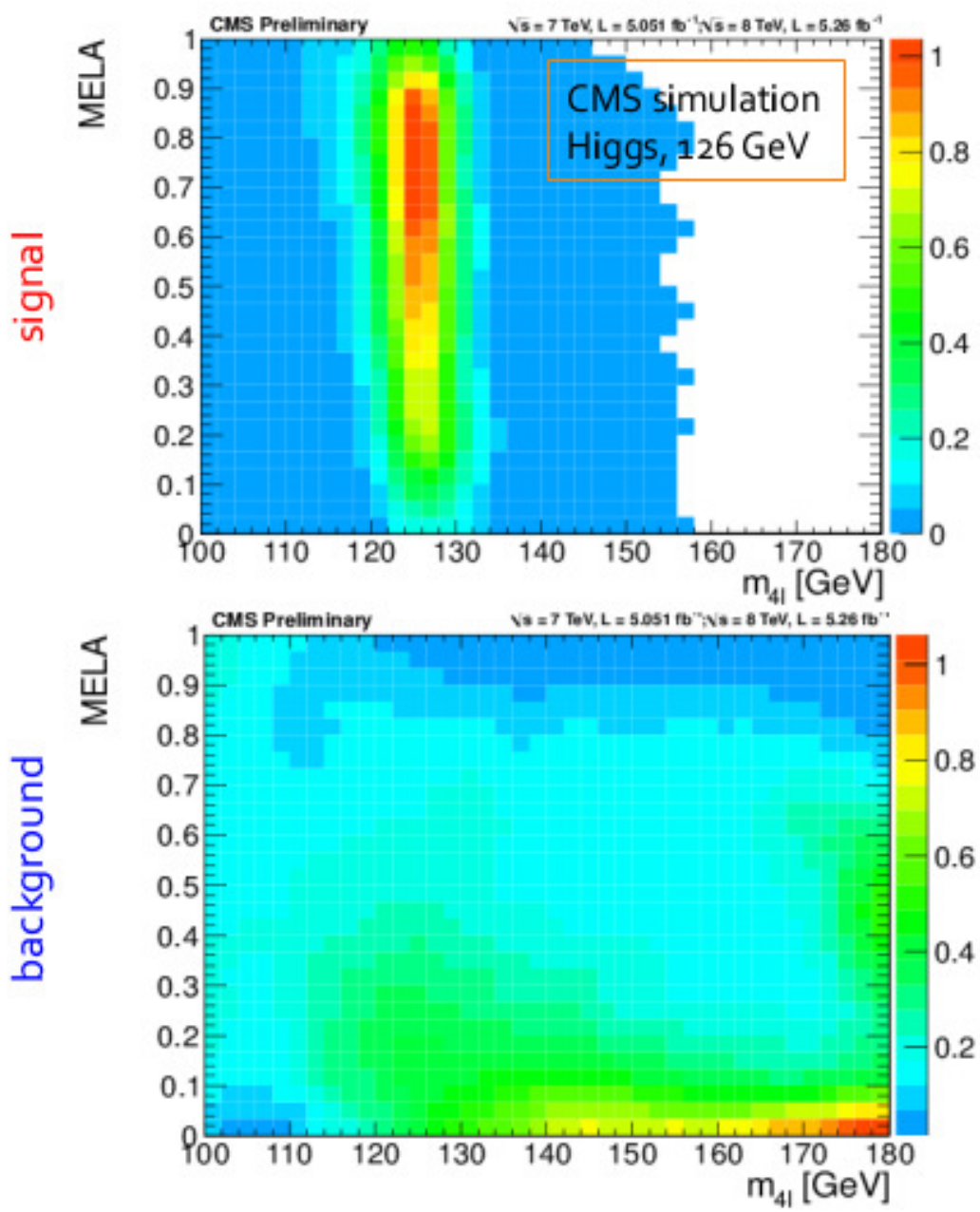

Fig. 28. Monte Carlo simulations of the signal and backgrounds for the $Z+Z^{*}$ final state. The matrix element likelihood analysis (MELA) variable is shown as a function of the $H$ mass.

is balanced by the smaller branching fraction with the consequent smaller number of expected $H$ events. In addition, in these decays a complete set of kinematic variables is available and all the kinematic correlations, for example the relative orientation of the two decay planes, are available to suppress the backgrounds. That information is encoded by CMS in the MELA variable and the distribution of MELA for an $H(125)$ compared to the backgrounds is shown in Fig. 28. A selection on the MELA will obviously enhance the signal to background ratio substantially.

The mass spectrum for $Z+Z^{*}$ is shown in Fig. 29, for a simple selection and also for a more discriminating selection using the MELA variable. There is a clear 


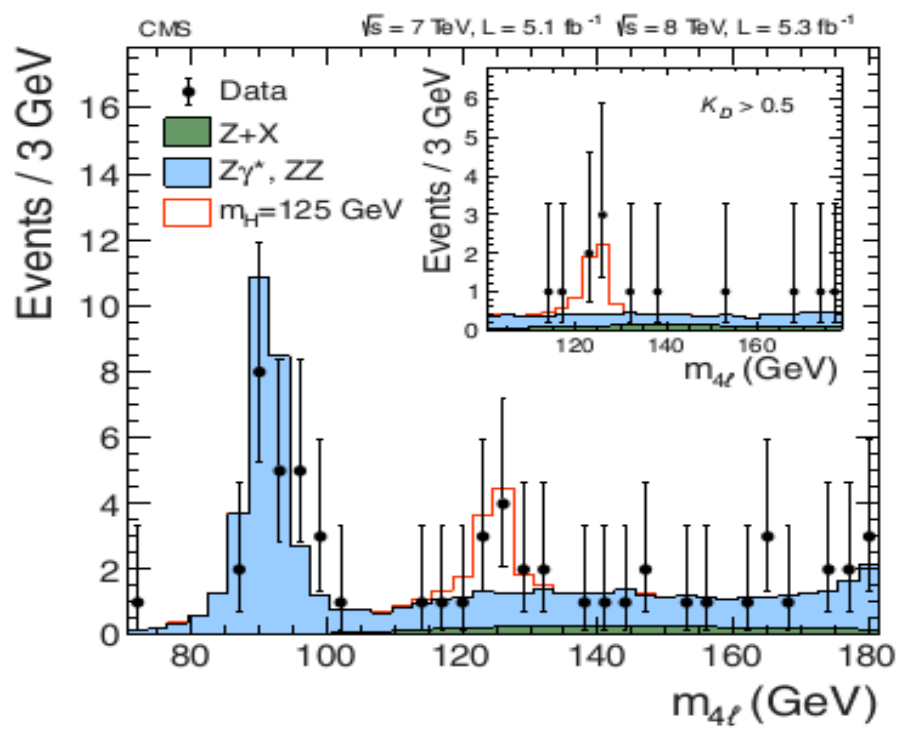

Fig. 29. Mass spectrum of the four-lepton final state. The insert has an additional requirement on the MELA variable.

clustering of events at a mass near $125 \mathrm{GeV}$ which gives support to the observed resonance in the di-photon mass spectrum. It is interesting to note that the fourlepton state has an available "standard candle." The $Z$ boson at $91 \mathrm{GeV}$ is evident in Fig. 29. The $Z$ resonance occurs because there are radiative $Z$ decays, Eq. (12), where the emitted photon decays virtually into a lepton pair. In this case the fourlepton invariant mass will be the $Z$ mass. Therefore, CMS is provided with a check on the four-lepton mass resolution and mass scale

$$
Z \rightarrow \mu^{+}+\mu^{-}+\gamma^{*} \rightarrow \mu^{+}+\mu^{-}+e^{+}+e^{-} .
$$

As was mentioned earlier, the expected number of $Z+Z^{*}$ events was about 20 before efficiency factors are applied. As seen in Fig. 29, there is an approximate agreement between the data and the predictions for an SM Higgs of $125 \mathrm{GeV}$ mass.

As was shown in Fig. 8, predictions for the $H$ branching ratio are that the $W+W^{*}$ final state provides a larger cross-section then does the di-photon or the $Z+Z^{*}$. However, the accessible $W$ decay modes with a sufficiently large signal to background are the leptonic decays, $W$ to lepton plus neutrino. Because the neutrinos are not measurable, the Higgs mass resolution in the $W+W^{*}$ search is not very precise. The assumed scalar nature of an SM Higgs, means that the mass of the final state dileptons tends to be low, as discussed later. The Higgs search in $W+W^{*}$ then uses the dilepton mass spectrum, shown in Fig. 30, in a shapedependent search. The major background in this search is continuum electroweak $W$ pair production. All the backgrounds must be well modeled because there is no sharp resonance structure in this final state. 


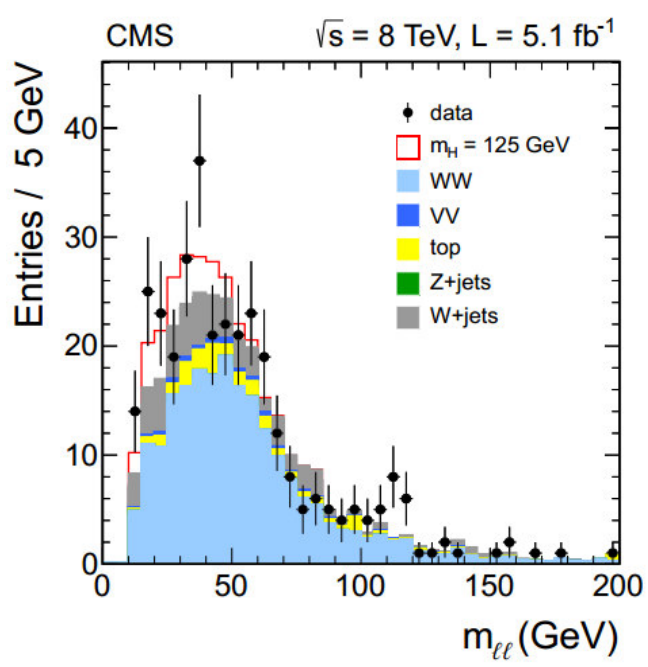

Fig. 30. Dilepton mass search in events with two leptons and MET. The $H$ signal at $125 \mathrm{GeV}$ is expected to populate the low end of the mass spectrum.

This final state is not as sensitive as the two previous ones. In order to see if the observed spectrum is statistically consistent with the production and decay of an SM Higgs, Monte Carlo models were used to simulate the "injection" of an $H$ signal into the simulated data to check the sensitivity of CMS in this particular final state. The results are shown in Fig. 31, where contours of confidence level at $68 \%$ and $95 \%$ are shown for the expected limits in the case of no Higgs $W+W^{*}$ decays and with decays at the full SM strength. The observed data limits are fully consistent with the SM $H$ prediction, although the most probable yield is somewhat less. As seen in Fig. 31, there is not a good mass discrimination in the $W+W^{*}$ decay mode. Nevertheless, the CMS results are consistent with an SM Higgs in this decay mode.

Data for the three final states are combined with less significant data on searches for $H$ decays into $b$ quark and tau lepton pairs and the extracted limits are given in Fig. 32 for low masses. The Higgs hypothesis is ruled out at the $95 \%$ confidence limit except for Higgs masses near $125 \mathrm{GeV}$. Recalling that the SM Higgs might have a mass anywhere from the LEP limit of $114 \mathrm{GeV}$ up to the limit set by unitarity of around one TeV, CMS have searched over the widest possible mass range and set the limits shown in Fig. 33. The expected and observed significances show that the signal at $125 \mathrm{GeV}$ is observed at about the expected level and that the CMS SM $H$ search is sensitive at a significance above two over a wide mass range.

At present a high mass Higgs is excluded for masses up to $600 \mathrm{GeV}$. That limit will be extended to about $1 \mathrm{TeV}$ with the full 2012 dataset, which will have data taking completed at the end of the 2012 calendar year. The resonance at $125 \mathrm{GeV}$ has a five standard deviation significance, which is sufficiently strong statistically to allow CMS to announce the discovery of a new particle. 


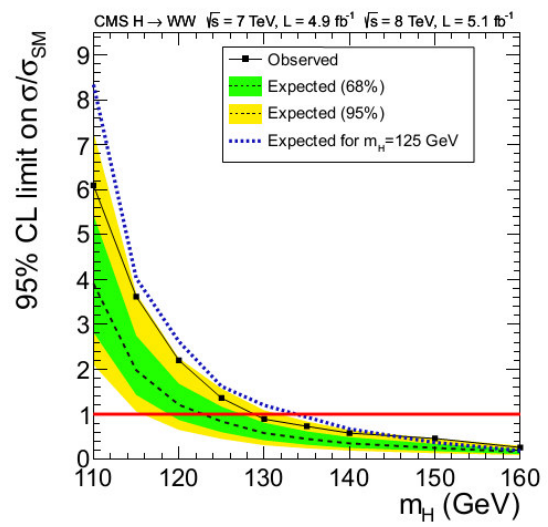

Fig. 31. Contours of $68 \%$ and $95 \%$ confidence limits as a function of the Higgs mass expected in the $W+W^{*}$ final state for production cross-sections expressed as a ratio to the calculated SM rate for the case with and without an SM $H(125)$ and the observed limit.

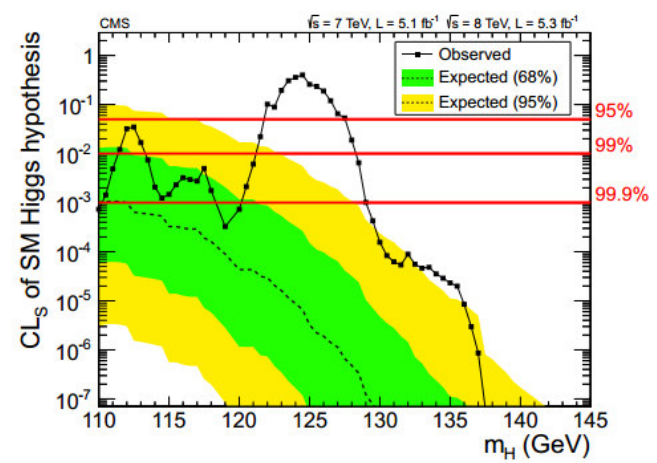

Fig. 32. Confidence level for the SM Higgs hypothesis for low Higgs masses in CMS. An SM Higgs is excluded at the $95 \%$ confidence level down to the LEP mass limit except for the mass region near $125 \mathrm{GeV}$.

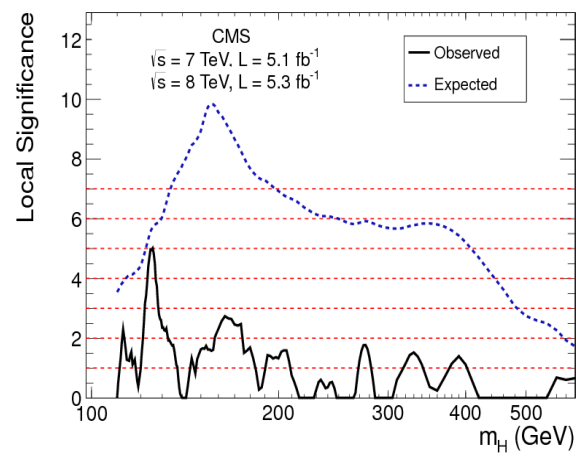

Fig. 33. Local significance for combined decay modes for an SM Higgs as a function of $H$ mass. The only significant signal appears at $125 \mathrm{GeV}$ and has a local significance of five. No other significant signal appears for SM $H$ masses up to about $600 \mathrm{GeV}$. 


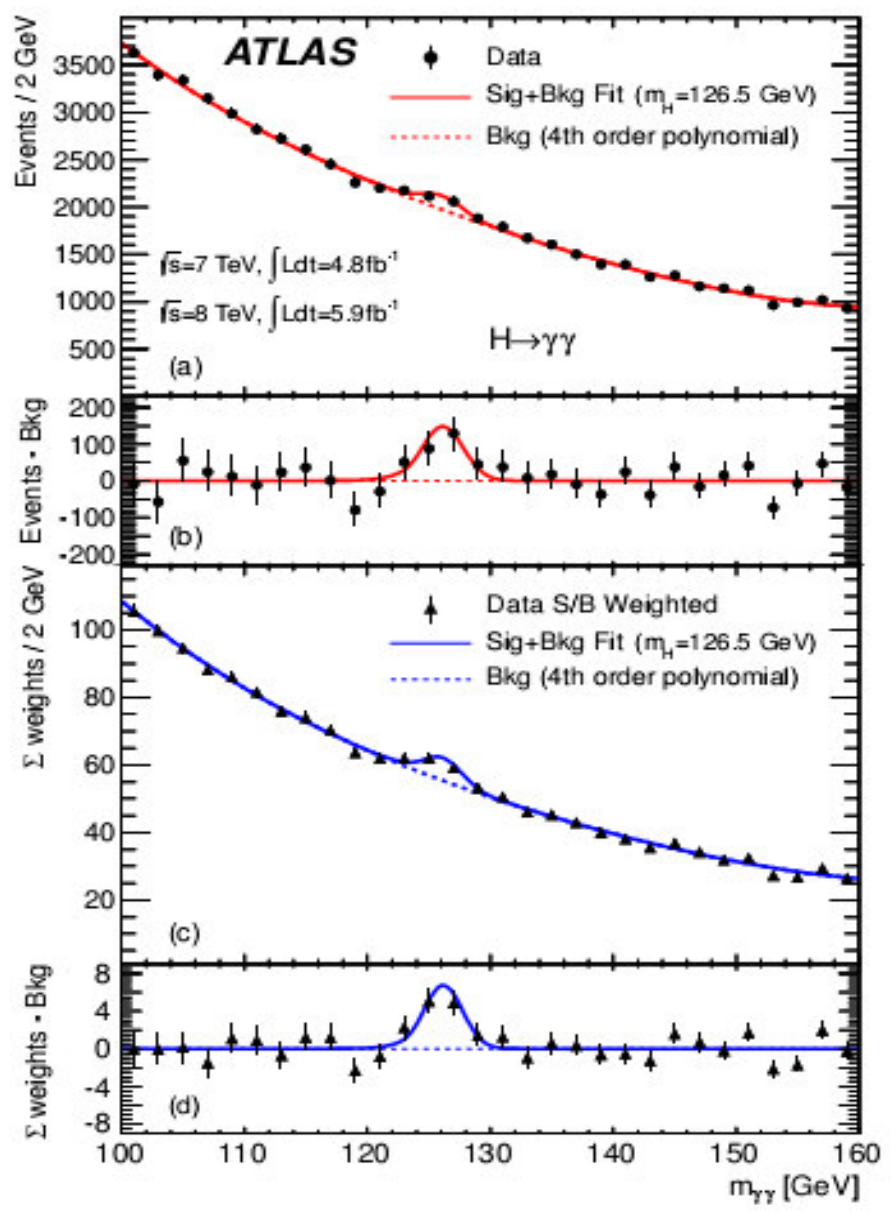

Fig. 34. The di-photon spectrum in the ATLAS experiment. The significance of the signal in this channel alone is above a local significance value of five.

There are two general purpose detectors in operation at the LHC, CMS and ATLAS. It is of crucial importance to have a statistically independent confirmation of the CMS result from the ATLAS data analyses. The di-photon spectrum measured in ATLAS is shown in Fig. 34. A highly significant resonant signal consisting of a few hundred events above background is also seen at the same mass as in CMS, which lends crucial support to the conclusion that a new particle, coupled to vector bosons, photons, $Z$ and $W$, has been discovered which has a mass of $125 \mathrm{GeV}$ and a width less than $1 \%$ of its mass.

Having established the signal, CMS have also looked at $H$ decays into leptons. Because the $H$ couples to mass, it will also decay preferentially into the heaviest pair of quarks (b) and leptons (tau) which is above threshold. Combining all five final states explored by CMS and assuming SM branching fractions, the best fit to 


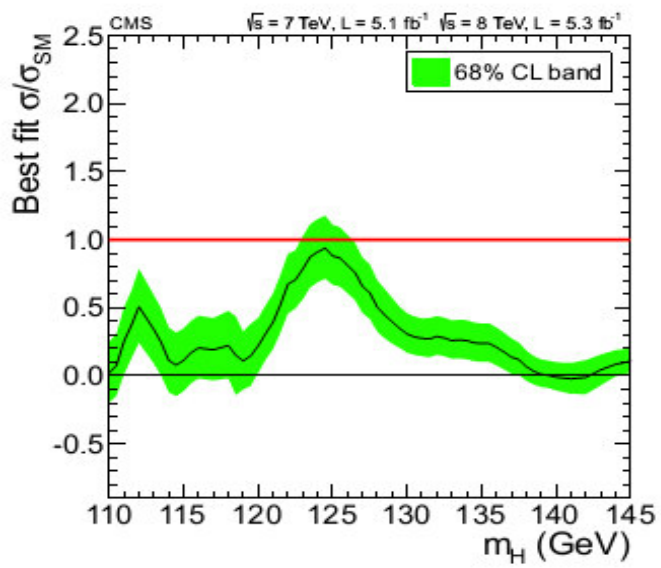

Fig. 35. Best fit to the cross-section normalized to an SM $H$ value as a function of the resonant mass using all the final states explored by CMS.

the overall cross-section as a function of the resonant mass is shown in Fig. 35. The particle at $125 \mathrm{GeV}$ has a cross-section, under these assumptions which is slightly less than that for an SM Higgs, although statistically consistent with the SM value. The local significances for the five final states are shown in Fig. 36 as a function of the $H$ mass. The overall significance is five and the di-photon has the most significance of the signals, followed by the $Z+Z^{*}$ which occurs at the same mass. The other final states are not as significant and they also do not have the same quality of good mass resolution.

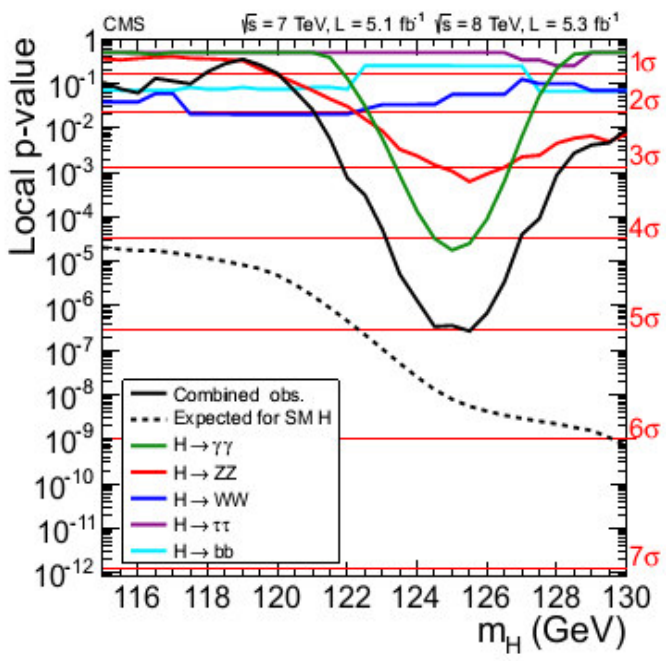

Fig. 36. Local probability as a function of resonant mass for the five final states analyzed by CMS. The di-photon and $Z+Z^{*}$ final states are the major contributors to the overall significance. 


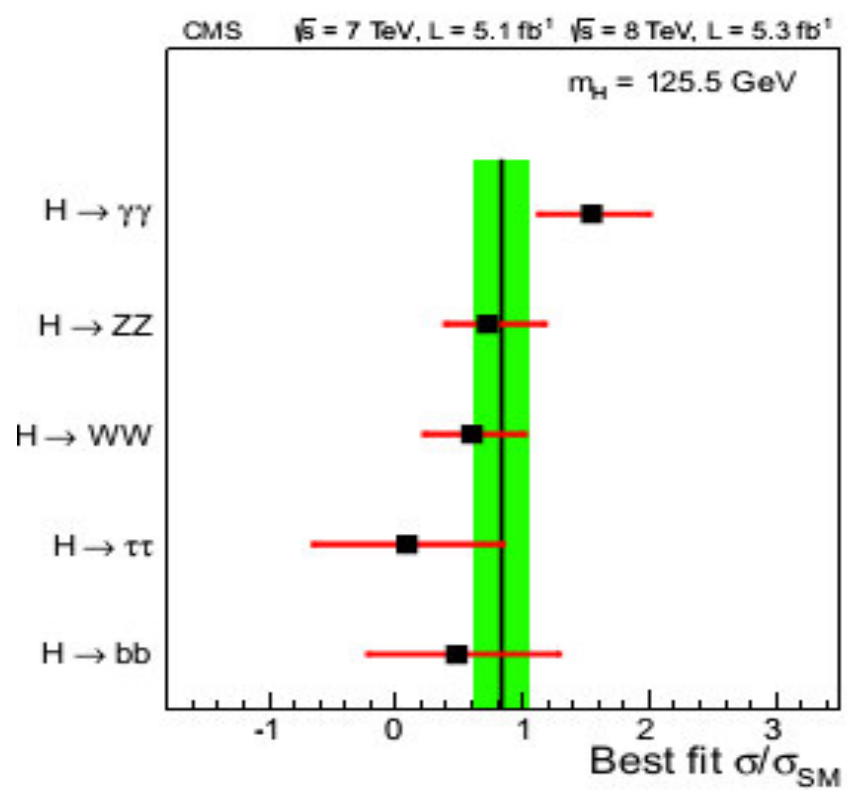

Fig. 37. Best fit values, with $68 \%$ errors shown, for the cross-sections in the five final states studied by CMS assuming SM branching fractions.

The best fit values for a cross-section assuming an SM decay branching ratio into one of the five final states studied in CMS are shown in Fig. 37. All are statistically consistent with the expected values for an SM Higgs. Nevertheless, the di-photon yield is somewhat larger than expected, the $Z+Z^{*}$ is fully consistent with expectations and the other three final states are all below expectations. It will be of great interest to see how these cross-section values evolve as more data becomes available, because an SM Higgs has values that are completely specified now that the mass is determined. Any significant deviation from the predictions of an SM $H$ branching fraction would then indicate new physics processes at play.

As shown schematically in Fig. 38, the Higgs coupling to the gluons and the photons, since they are massless, is not a direct three-body coupling as it is for the $W, Z$ and the quarks and leptons. It should be mentioned that the largest production mechanism for an SM Higgs is the fusion of gluons into virtual quark pairs which then inversely decay virtually into the Higgs boson, called ggF. If this process were absent, the cross-section, which was observed to be compatible with the SM value, would likely be somewhat smaller. Therefore, it seems likely that the observed particle does couple fairly strongly to top quarks, but a direct measurement, such as the observation of a final state of associated production of an $H$ and top quark pairs will be very informative.

For the ggF production process, the largest coupling is to the virtual top pairs while for the di-photon decay the virtual particles are the charged, massive $W$ and top. Any new particles in a virtual loop might enhance the di-photon width of 

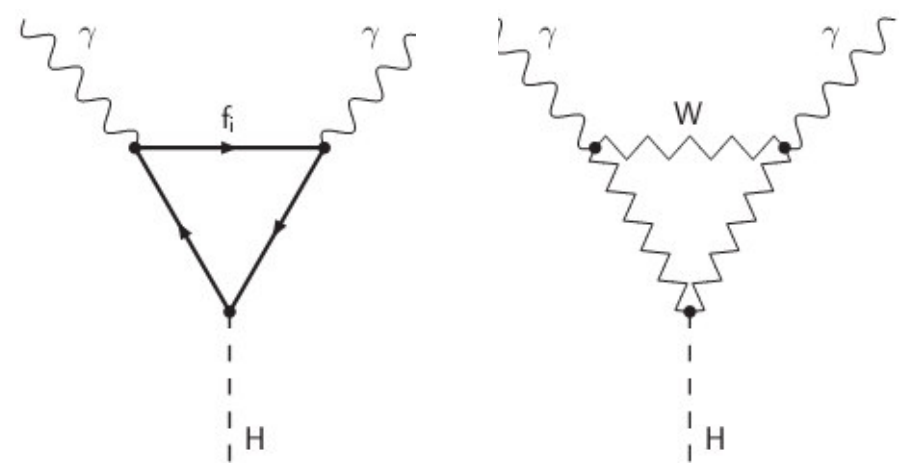

Fig. 38. Loop diagrams for SM $H$ coupling to di-photons. The two main contributions are intermediate $W$ bosons and top quarks in the SM. Strong $H$ production is similar and occurs with gluons creating a top quark loop which "fuses" into an SM $H$ or ggF.

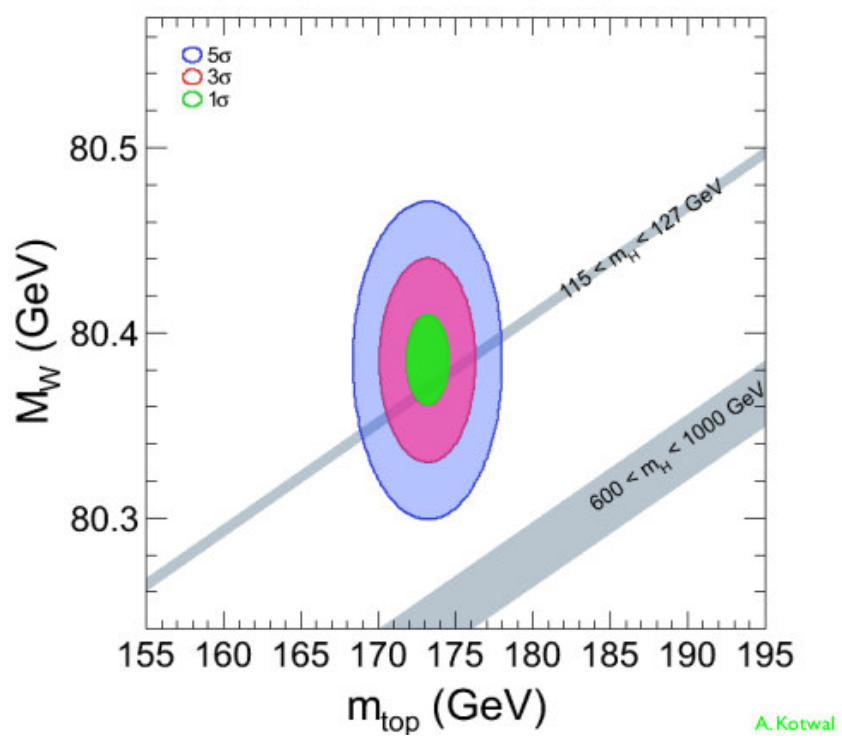

Fig. 39. Tevatron results for precision measurements of the top and $W$ mass. The SM expected region for a Higgs mass of about $125 \mathrm{GeV}$ is overlaid and is clearly consistent with the prediction.

a non-SM Higgs which makes the more precise measurements of the couplings a pressing issue.

The Tevatron at Fermilab stopped taking data at the end of 2011. A major legacy of the Tevatron is the very precise measurements of the top and $W$ masses ${ }^{63}$ particularly because they constrain possible Higgs masses since the top propagator contains the effect of a virtual emission and absorption of an $H$ boson. The final Tevatron results for these masses are shown in Fig. 39. The observed particle at $125 \mathrm{GeV}$ is clearly compatible with the SM predictions for the Higgs. 


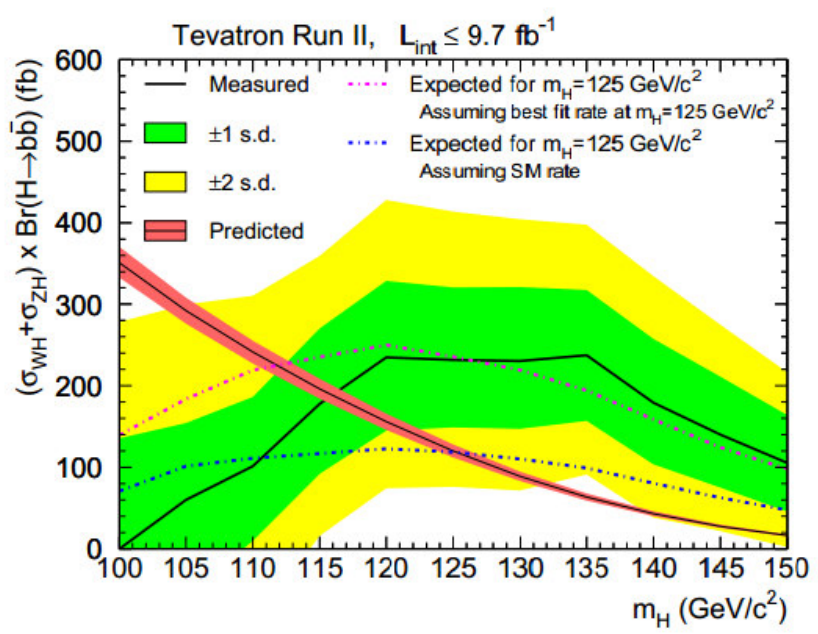

Fig. 40. Results from CDF and D0 on the limits for the $b$ quark pair decay of an SM Higgs as a function of the $H$ mass.

The Tevatron experiments have also made direct searches for the Higgs. ${ }^{62}$ Their focus has been on the associated production of an $H$ with a $W$ or $Z$ with the $H$ then decaying into a $b$ quark pair which is the most sensitive decay mode for the Tevatron. The combined result for a limit on $b$ quark pair decays of an SM Higgs is displayed in Fig. 40. The results are consistent with a Higgs having a mass of $125 \mathrm{GeV}$, and support the CMS and ATLAS results, but at a statistical local confidence level of only three, not sufficient to claim a discovery. More data from the LHC from the 2012 data run should shed additional light on the coupling of the $125 \mathrm{GeV}$ particle to leptons and quarks.

\section{The Future}

At design operation, there are about 1 billion interactions per second with initial state energy of $14 \mathrm{TeV}$ in the CM. The LHC was designed to operate with integrated luminosities of $100 \mathrm{fb}^{-1}$ per year for 10 years. In order to decisively study the $1 \mathrm{TeV}$ mass scale, it is therefore necessary to examine a total of 100,000 trillion interactions. The LHC and the experiments are, near the end of 2012, only $3 \%$ of the way there and only at $\frac{1}{2}$ the design energy. The LHC enterprise is just starting a long voyage of discovery into the future.

The mass of the new state can be determined for each of the final states with a robust signal and with a good mass resolution. In Fig. 41, the masses and $68 \%$ likelihood contours are shown for two varieties of di-photon final states and for the $Z+Z^{*}$ final state. The combined results for mass are about $125 \mathrm{GeV}$ with an error of $1 \mathrm{GeV}$. Given the CMS mass resolution as determined from the data on the $Z$ mass spectrum, the intrinsic width of the $H$ is less than about $1 \mathrm{GeV}$ while an SM Higgs would have a $4 \mathrm{MeV}$ width. 


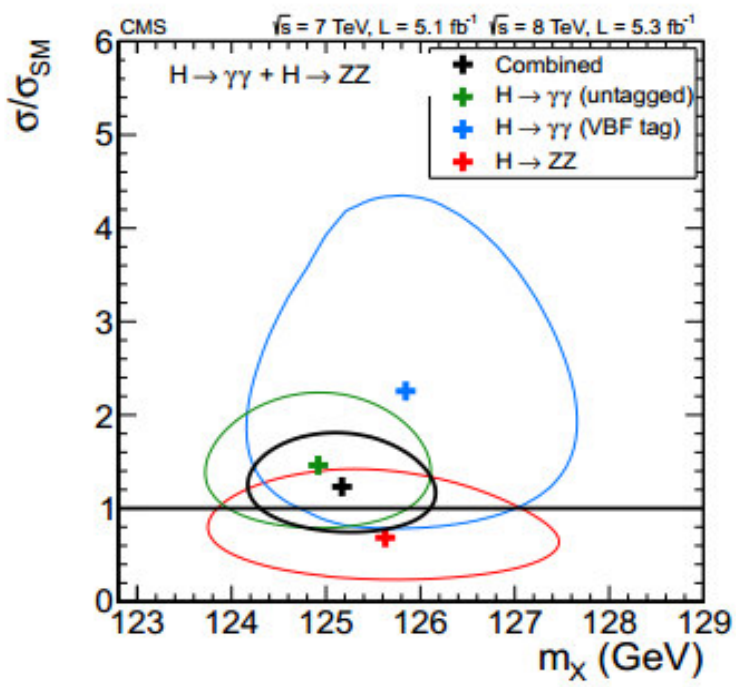

Fig. 41. Mass determination of the resonance discovered at CMS for three of the final states which were explored.

Having discovered a new particle the next steps are to determine its quantum numbers. Decay into a di-photon means that the spin of the parent could be 0,1 or 2. First, there is Yang's theorem ${ }^{64}$ which states that a spin one state cannot decay into two photons. The theorem is easiest to visualize in the rest frame of the parent particle, where the decay photons have momenta $\mathbf{k}$ and polarization vectors $\varepsilon$. It is impossible to construct a decay amplitude, $A$, which is Bose symmetric, for example the amplitude in Eq. (13) is not boson exchange symmetric

$$
A \sim\left(\varepsilon_{1} \times \varepsilon_{2}\right) \cdot \varepsilon_{H} .
$$

As regards the spin, the previously mentioned $W+W^{*}$ analysis (Fig. 30) relies on the assumed scalar nature of the SM Higgs boson. The spin correlations for a spin zero state which decays into $W$ pairs is shown in Fig. 42. The scalar $H$ must decay into oppositely directed $W$ bosons with oppositely directed spins. The subsequent $(V-A)$ structure of the weak interactions makes particles (electrons) left-handed and antiparticles (positrons) right-handed. That lepton spin alignment means that

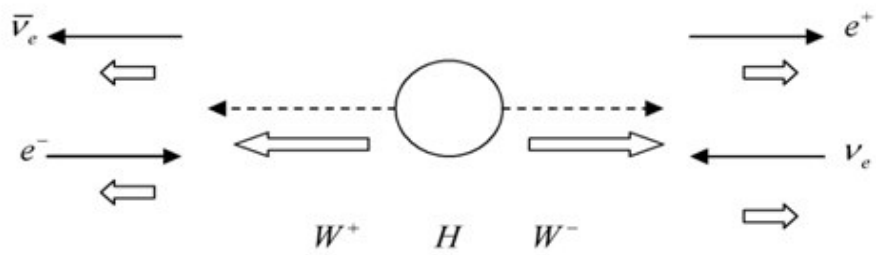

Fig. 42. Spin correlations in the decay of a scalar parent particle into $W$ pairs with subsequent two-body leptonic decays. 
the charged lepton pair is emitted preferentially collinear and thus with low mass. By the end of 2012, CMS should be able to make a statement at $95 \%$ confidence level about whether the new particle has spin zero or spin two.

The parity of the state can also be determined, in this case most easily by measuring the full decay chain in $Z+Z^{*}$ to four charged leptons. This decay is preferred to $W+W^{*}$ because the neutrinos are unmeasurable in $W$ leptonic decays. For positive or negative parity the polarization vectors of the $Z$ are oriented differently, and that polarization is encoded in the decay planes of the two pairs of leptons, which act as polarization analyzers. This is not a new technique. It was used 60 years ago in the determination of the neutral pion parity. ${ }^{65}$ In the decay $\pi^{0} \rightarrow \gamma+\gamma \rightarrow\left(e^{+}+e^{-}\right)+\left(e^{+}+e^{-}\right)$a scalar pion would tend to have aligned decay planes (Eq. (14)), while a pseudoscalar would tend to have orthogonal orientation of the planes. This technique was more recently used in the decay, $\eta_{c} \rightarrow \phi+\phi \rightarrow K^{+}+K^{-}+K^{+}+K^{-}$(Ref. 66) to determine the parity of that charmonium state

$$
\varepsilon_{1} \cdot \varepsilon_{2}, \quad\left(\varepsilon_{1} \times \varepsilon_{2}\right) \cdot \mathbf{k} .
$$

For the Higgs boson a complete methodology has been developed ${ }^{67}$ and techniques which are being applied in CMS have been developed, ${ }^{68}$ which utilize the complete set of kinematic variables. The distribution of the log of the likelihood ratio for the scalar and pseudoscalar hypotheses is shown in Fig. 43 for the $Z+Z^{*}$ decay mode. CMS should have sufficient data logged by the end of 2012 to make a $95 \%$ confidence level statement about the parity of the new particle.

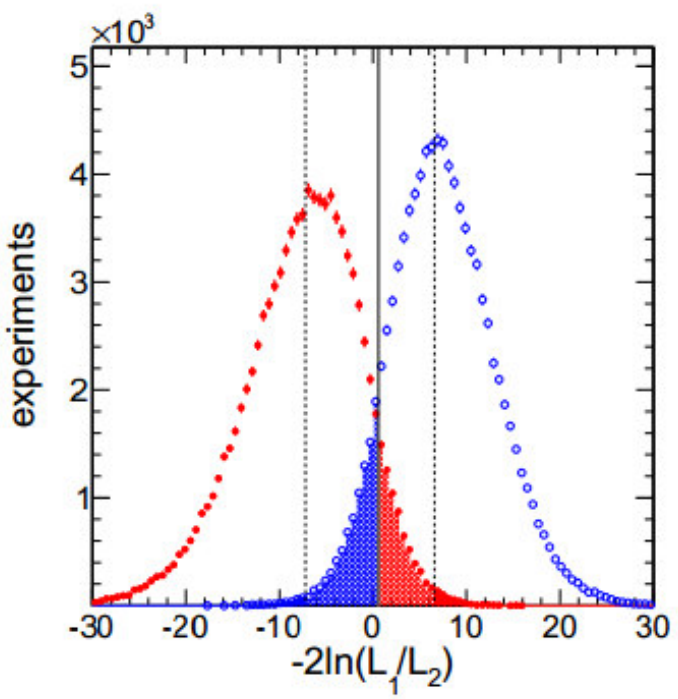

Fig. 43. (Color online) Log of the likelihood ratio for scalar (red) and pseudoscalar (blue) hypotheses in $Z+Z^{*}$ decays of an SM Higgs boson when the local statistics is at the level of 5 for the Higgs hypothesis itself. 68 


\section{CMS Projection}

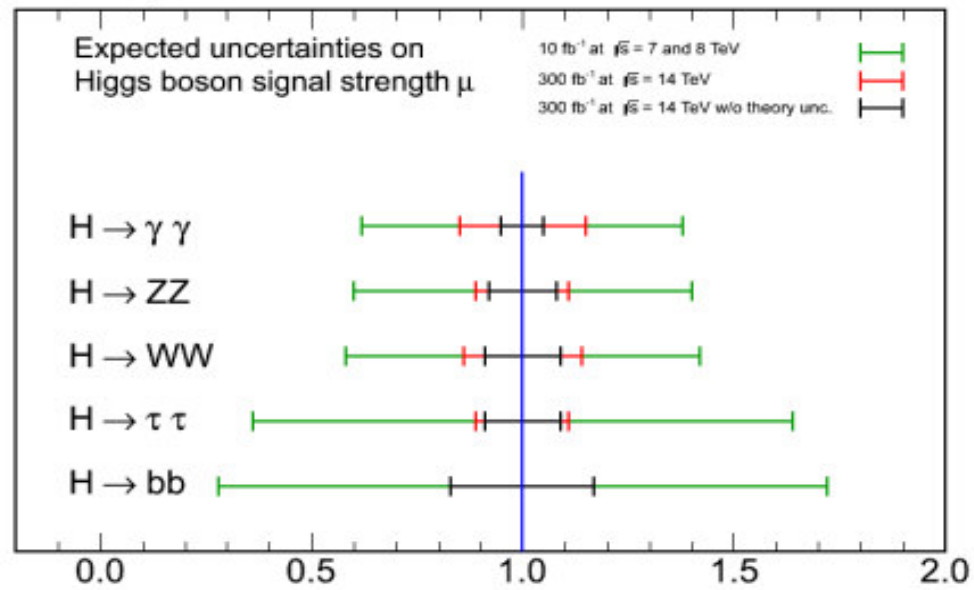

Fig. 44. (Color online) Projected accuracy of the coupling of the new particle for five final states with respect to the SM coupling for the present (green) and for 30 times more data at $14 \mathrm{TeV}$ $\mathrm{CM}$ energy, with and without theoretical uncertainties.

The couplings of an SM Higgs boson to all the other SM particles are uniquely determined once the mass is known. Therefore, it is quite important to press ahead on a more accurate determination of those couplings in the future. A projected estimate of the accuracy of the determination by CMS of the couplings is shown in Fig. 44. The time scale for the LHC is that it will be off for repairs in 2013 and 2014. Starting in 2015 operation at design energy of $14 \mathrm{TeV}$ and design luminosity of $100 \mathrm{fb}^{-1}$ per year will begin, evolving to higher luminosity with time. The projected accuracy of $10 \%$ to $15 \%$ for the most accurately known decay modes which are shown in Fig. 44 is then expected to be available at the end of 2017.

The SM Higgs boson has not only to give masses to the SM particles but it must make the scattering of vector boson pairs unitary. As a first step in exploring the self-couplings of the electroweak bosons, the triple vector vertices can be explored by studying the production of a pair of vector bosons, as seen in Fig. 45, where the leading order Feynman diagrams are shown. There are " $t$-channel" quark exchange diagrams, but in addition there is a triple gauge vertex. Triple and quartic couplings are intrinsic to gauge theories and lead to nonlinear dynamics. Fundamentally that is due to the fact that the $H, W, Z$ and gluons all carry the "charge" of their interaction. In contrast, the photon has no charge and electrodynamics is a linear theory.

CMS have begun ${ }^{69}$ the study of these triple gauge couplings (TGC) and searched for anomalous TGC (aTGC) where current limits are plotted in Fig. 46. Similar limits were found and LEP and at the Tevatron. However, by the end of the 2012 data taking the LHC experiments should set the world's best limits on aTGC and further elucidate whether the electroweak couplings are as predicted by the SM. 
D. Green
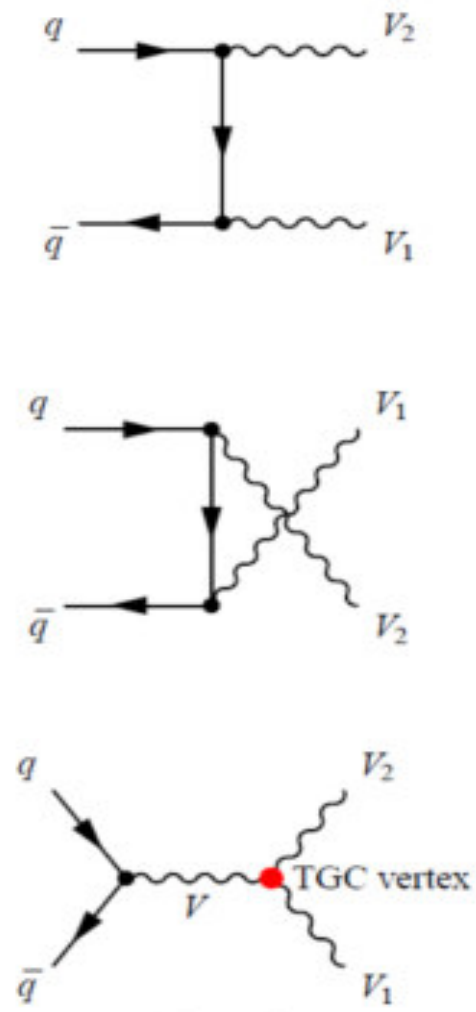

Fig. 45. Feynman diagrams for the production of a pair of electroweak vector bosons.

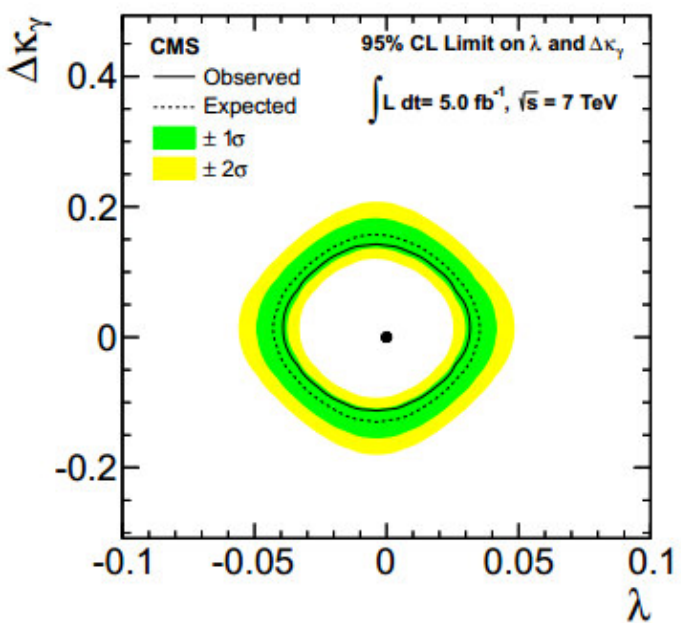

Fig. 46. Limits on aTGC in CMS from the production dynamics of $W$ pairs. 


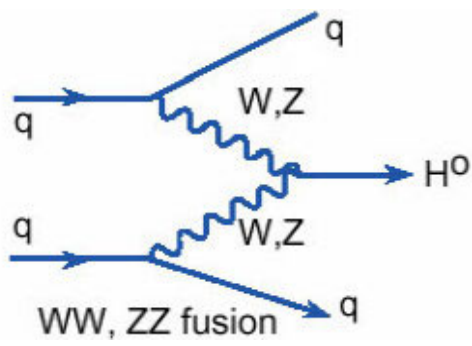

Fig. 47. Feynman diagram for quarks radiating virtual $W$ and $Z$ bosons that then scatter or fuse to produce an $H$ boson.

Continuing in the study of self-couplings, an SM Higgs boson must "unitarize" the scattering of the $W$ bosons. Without an SM Higgs the $S$ wave amplitude, Ao, grows as the CM energy squared, $\hat{s}$.

The Higgs mass must then be, Eq. (15), about one TeV or less, which sets the high mass $H$ limit which was quoted above. In many theories which go beyond the SM there are multiple Higgs-like bosons, so that a complete search over all masses and all available dynamics is mandatory

$$
A_{o}(W+W \rightarrow W+W) \sim \frac{3 \alpha_{W} \hat{s}}{16 M_{W}^{2}}<1, \quad M_{H}<\sqrt{\hat{s}}<\frac{4 M_{W}}{\sqrt{3 \alpha_{W}}} \sim 1 \mathrm{TeV} .
$$

The isolation of the $W+W$ scattering process in $p-p$ collisions is easiest in the "vector boson fusion" or VBF process. This process is the analogue of the gammagamma fusion in electron-positron collisions and it begins to compete as the dominant process when the incoming quarks become highly relativistic because in that case their radiation becomes dominant. As seen in Fig. 7, in the case of $p-p$ collisions (fundamental gluon-gluon and quark-quark collisions) the VBF process is equal to the ggF process at a mass of about $1 \mathrm{TeV}$. The VBF Feynman diagram for $H$ production is shown explicitly in Fig. 47. As can be seen from that diagram the VBF process can be fairly well isolated by requiring two quark jets which go far forward (backward) in the $p-p$ CM frame and which have the $W+W$ as determined from their decay products in the region between these "tag jets" in angle.

The ggF process works through a virtual top pair with a resulting loop integral indicted by $I$ and with two powers of the strong coupling constant from the gluon to top transition. In contrast the VBF process has two powers of the weak coupling constant from the virtual emission of the two vector bosons in addition to kinematic factors due to the radiative spectrum of the emitted bosons yielding the factor $\tau=M^{2} / \hat{s}$ which enhances VBF at large sub energies, $\sqrt{\hat{s}}$, and makes plausible, Eq. (16), that VBF can become important at high masses

$$
\begin{aligned}
\sigma(g g \rightarrow H) & \sim \frac{\pi^{2} \Gamma(H \rightarrow g g)}{M^{3}}=\frac{\left[\alpha_{W} \alpha_{s}^{2}|I|^{2}\right]}{\left(72 M_{W}^{2}\right)}, \\
\frac{\sigma(g g \rightarrow H)}{\sigma(q \bar{q} \rightarrow q \bar{q} H)} & \sim \frac{\left[\left(\alpha_{s} / \alpha_{W}\right)^{2}\left|I^{\prime}\right|^{2}\right]}{4 \ln (1 / \tau)} .
\end{aligned}
$$




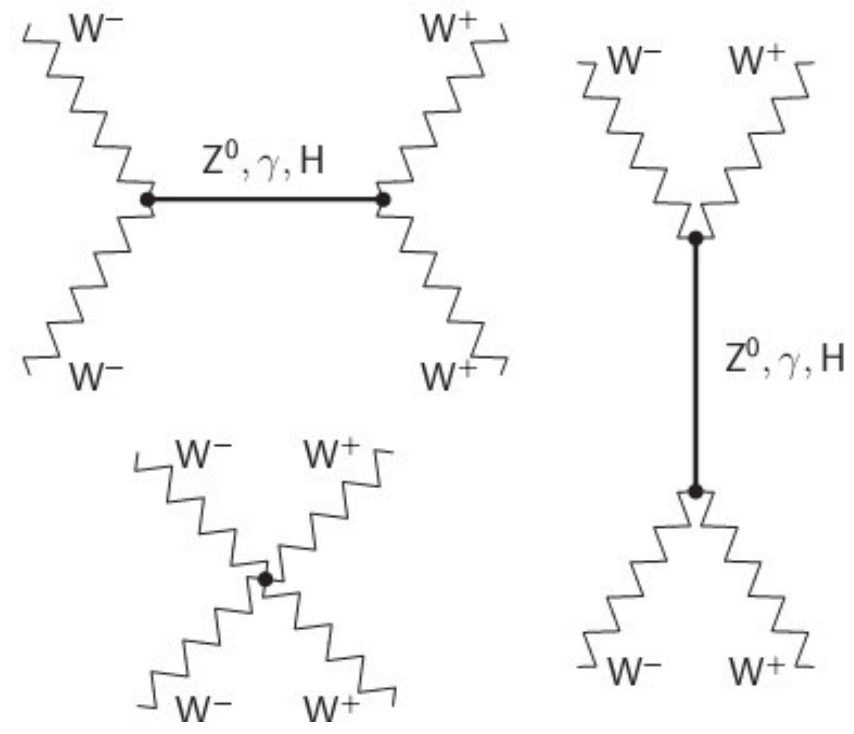

Fig. 48. Feynman diagrams for $W+W$ scattering in the SM with the addition of an SM Higgs boson.

The Feynman diagrams at leading order for $W+W$ scattering are shown in Fig. 48. They are the SM $s$ - and $t$-channel photon $(A)$ and $Z$ diagrams plus the quartic four $W$ electroweak coupling. These diagrams alone violate unitarity and are made unitary by the addition of two diagrams, the $s$ - and $t$-channel Higgs $(H)$ diagrams in the SM.

Clearly, isolating the VBF process using the tag jets and extracting the $W+W$ scattering gives new tests for the SM. First anomalous quartic couplings can be studied. The production by ggF of three $W$ bosons is also sensitive to quartic couplings, but there are other diagrams that dilute the sensitivity. Finally, if the new particle is the SM $H$, then the $W+W$ scattering is unitary at all $W W$ masses and the VBF process will allow that test to be made.

It may turn out that the new particle does not full unitarize $W+W$ scattering. In any case, future study of $W+W$ scattering is crucial to the establishment, or not, of the new particle as the SM Higgs boson. A possible alternative ${ }^{70}$ to the SM $H$ is shown in Fig. 49. In this case the scattering is only partially made unitary, the degree of which is set by a parameter which is varied in Fig. 49. By examination of the figure, it seems clear that a close inspection of $W+W$ scattering over the full mass range up to about one $\mathrm{TeV}$ should be performed when the statistics sufficient to do so become available.

In 2015 and later the LHC will operate at the full $14 \mathrm{TeV}$ CM energy and with enhanced luminosity. This enhanced operation will lead to major increases in the parton luminosity at high mass scales. In Fig. 50, the gluon-gluon and quark-quark parton luminosities ${ }^{71}$ as a function of the produced mass are plotted for seven $\mathrm{TeV}$ 


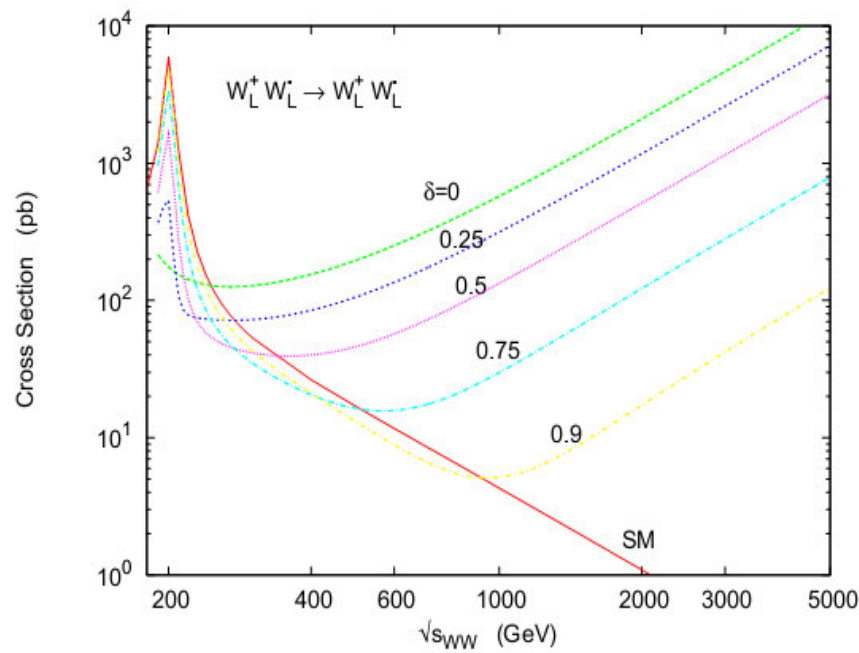

Fig. 49. $W+W$ mass spectrum for a model where the new state only partially makes the scattering unitary. Deviations from the SM can occur at high $W$ pair masses.

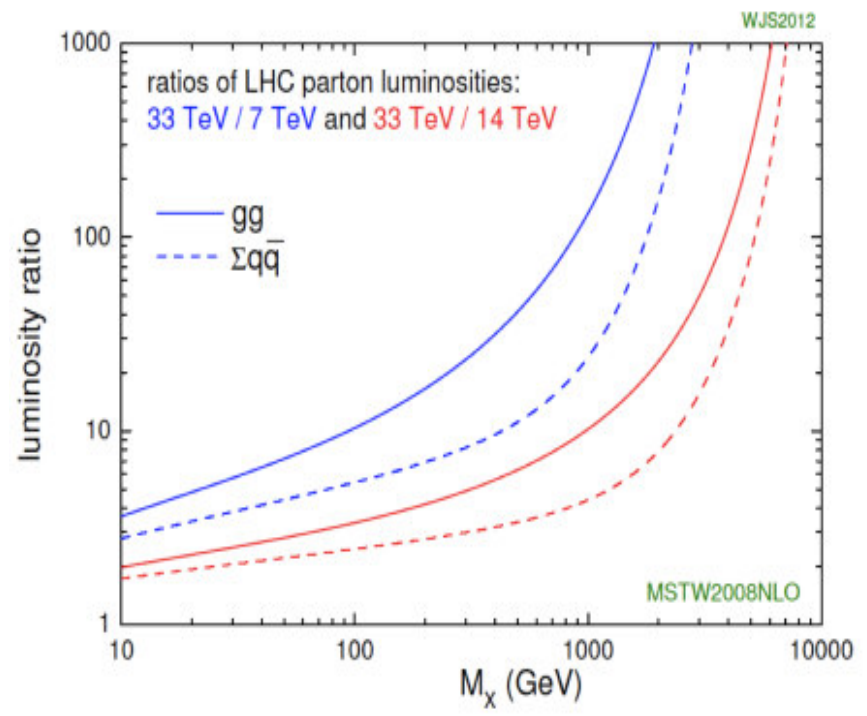

Fig. 50. Parton luminosity ratios for 7, 14 and $33 \mathrm{GeV}$ in $g-g$ and $q-q$ interactions for the production of different mass states.

(2011 running) with respect to $14 \mathrm{TeV}$ (2015 running) and $33 \mathrm{TeV}$ (a future possible upgrade path for the LHC accelerator). The gains that a search for high mass states make are very clear; a factor of at least one hundred comparing 7 and $33 \mathrm{TeV}$ in the most unfavorable $(g-g)$ case at a mass scale of only one $\mathrm{TeV}$. The gains at higher mass scales rise very rapidly with mass. 


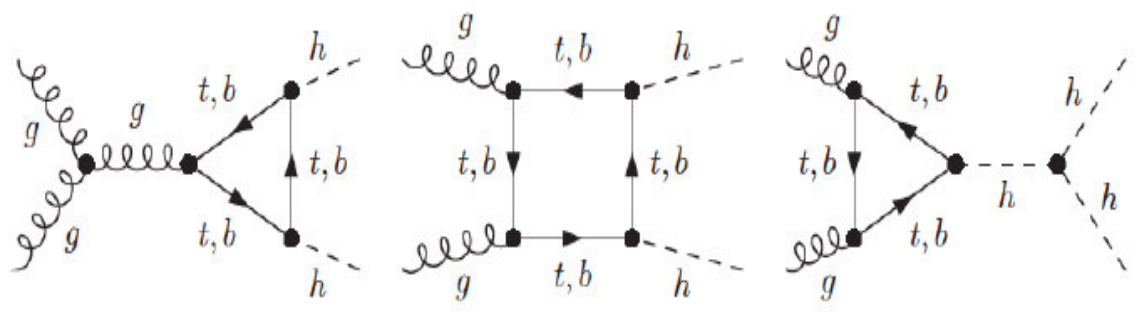

Fig. 51. Feynman diagrams for the ggF production of a pair of $H$ bosons. Virtual top loops are the most prominent contributors.

Continuing the examination of whether the new particle is the SM Higgs, an examination of the self-couplings of the particle is needed because the SM $H$ triple and quartic self-couplings are completely specified in the SM. The most favorable test is to produce a pair of $H$ bosons. The Feynman diagrams for that process are shown in Fig. 51. Note that the cross-section for this process ${ }^{72-74}$ is much smaller than that for a single $H$ boson. Therefore, the improved energy and luminosity planned for future LHC running will be needed to be able to make this test of the new particle. The ability to explore the quartic couplings of the Higgs boson appears to be too difficult even after these upgrades and improvements because of the extremely low value for the cross-section for the production of three $H$ bosons in an interaction.

Even if the new particle appears to be congruent with the SM Higgs, there remain problems. A fundamental scalar like the $H$ is very sensitive to quantum loop corrections to its mass. The spin $\frac{1}{2}$ fermions and the vector bosons are only changed logarithmically while the Higgs scalar is unique in the SM as the only scalar boson. The Higgs mass diverges quadratically with the mass cutoff scale which, presumably, comes from higher mass scales, such as the Planck or Grand Unified (GUT) scales. In that case the Higgs mass is raised nearly to that scale, $\Lambda$. The major $H$ mass changes due to loop couplings to $W, Z, H$ itself (triple coupling) and top are shown in Eq. (17)

$$
\delta M_{H}^{2}=\frac{\alpha_{W}}{8 \pi}\left(\frac{\Lambda}{M_{W}}\right)^{2}\left(6 M_{W}^{2}+3 M_{Z}^{2}+M_{H}^{2}-12 M_{t}^{2}\right) .
$$

To avoid this "hierarchy problem" several solutions have been proposed. One solution is to assert that the new particle is not a fundamental scalar but rather a fermion pair bound state. This is reminiscent of the case when Cooper pairs supplanted the Landau-Ginzberg superconductivity field. That dynamical symmetry breaking solution requires a new binding force and, presumably, a new U(1) gauge boson. Limits on the cross-section times di-lepton branching ratio for CMS obtained with a $33 \mathrm{GeV}$ CM upgraded LHC are shown in Fig. 52.

Another possible solution is that there are extra dimensions so that the high mass scale is not the Planck scale but a scale around a few TeV and gravity only 


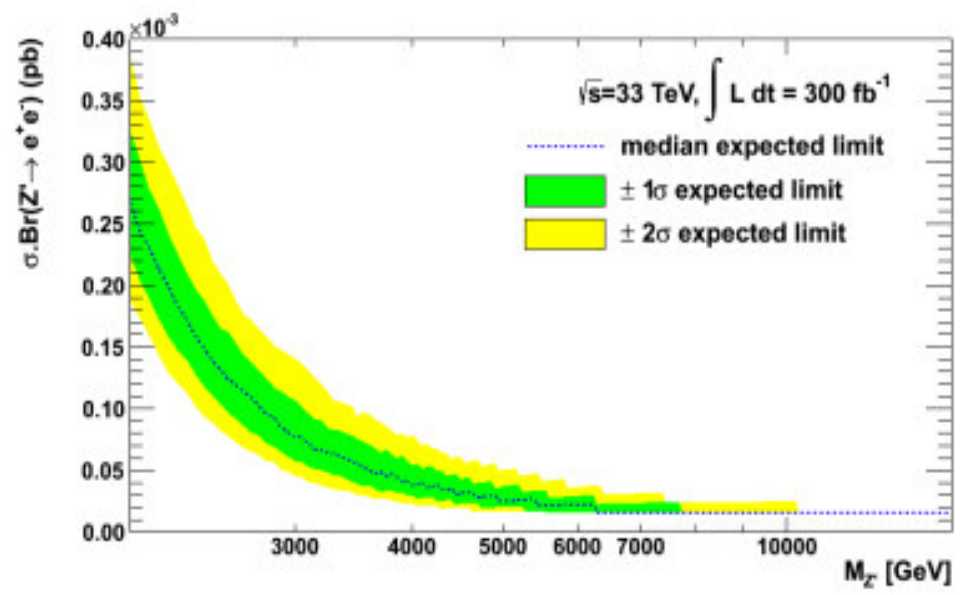

Fig. 52. Mass reach in CMS for an upgraded LHC accelerator operating at $33 \mathrm{TeV}$ for an integrated luminosity of $300 \mathrm{fb}^{-1}$ as a function of the mass of a new $\mathrm{U}(1)$ boson.

looks weak (i.e. high mass) because it leaks into the extra dimensions. In that case there is no hierarchy problem and the issue is to find evidence for these extradimensional states. The planned LHC upgrades will then sharpen the search for such states which is already in train in CMS.

A third possibility is that supersymmetry (SUSY) exists and cancels the loop contributions of the SM particles to the $H$ mass with SUSY partners. This only works if the partners are not too massive, of order a few $\mathrm{TeV}$. Therefore, using the upgraded LHC, CMS plans to explore this mass range in a search for TeV mass SUSY particles. A plot of the mass range for SUSY in various upgrade scenarios ${ }^{71}$ is shown in Fig. 53. It appears that such studies can establish or not the existence of SUSY for masses of a few $\mathrm{TeV}$ in these scenarios and therefore test whether SUSY is the solution of the hierarchy problem.

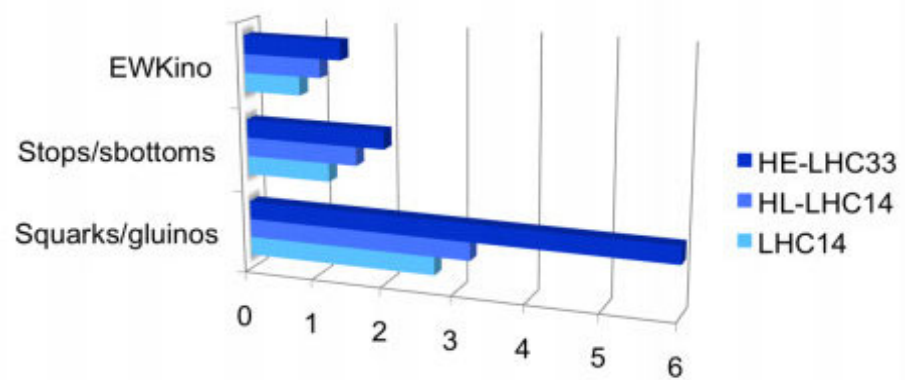

Fig. 53. Mass reach for SUSY particles of first quark generation, third generation and electroweak in three LHC configurations, LHC at $14 \mathrm{TeV}$, at $14 \mathrm{TeV}$ but $10 x$ luminosity and LHC at high energy, $33 \mathrm{TeV}$ and design luminosity. 


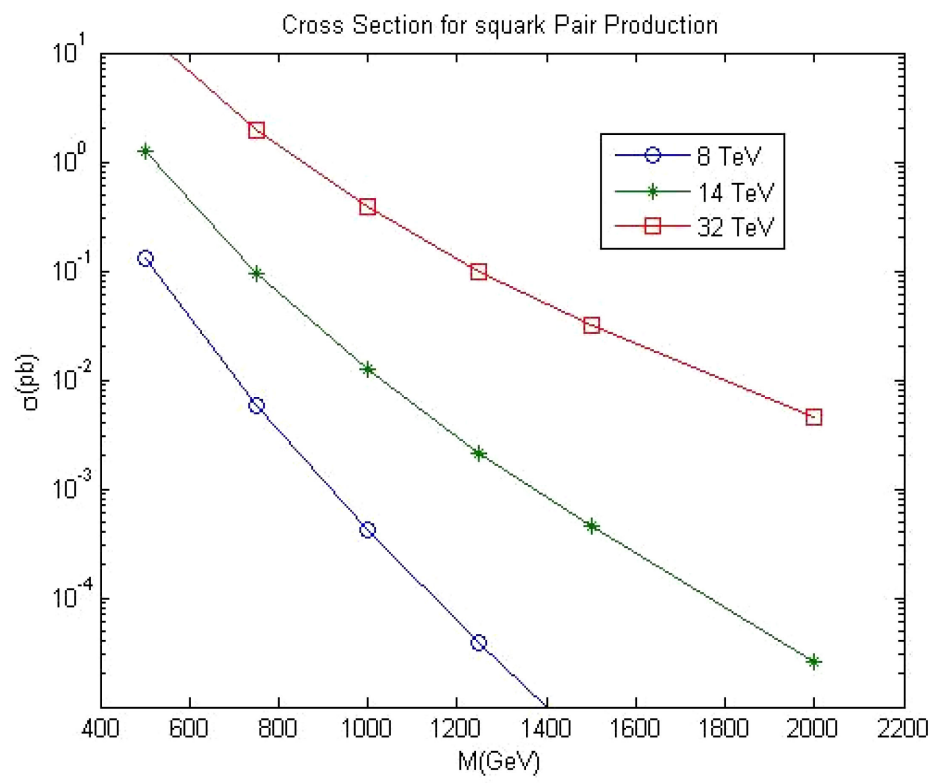

Fig. 54. Cross-section at leading order for the production of SUSY quarks (squarks) as a function of their mass for CM energies of 8,14 and $33 \mathrm{GeV}$.

In Fig. 54, is plotted the cross-sections for SUSY quark (squark) pair production at 8, 14 and $33 \mathrm{TeV}$ CM energy as a function of squark mass. At a squark mass of two $\mathrm{TeV}$ the ggF cross-section gain from $14 \mathrm{GeV}$ to $33 \mathrm{GeV}$ is a factor about 100 . Clearly, for these high mass explorations, the enhanced cross-section at $33 \mathrm{TeV}$ has a definitive advantage. This is also clear in Fig. 53, where the high energy reach for gluinos is twice that for a high luminosity variant; six compared to two TeV.

SUSY provides the extended SM with a dark model candidate which is lacking in the SM, the neutralino as the lightest SUSY particle. CMS has an extensive program to search for SUSY particle pair production. Additionally, CMS will continue to directly search for dark matter production at the LHC. Dark matter can be looked for independently of the SUSY hypothesis. In fact CMS have already published ${ }^{75}$ limits on dark matter, plotted in Fig. 55, which are the worlds best spin dependent limits at all masses and the best spin independent cross-section limits in the low mass region, less than about $10 \mathrm{GeV}$. CMS will continue these searches and set improved limits which become possible in the future with the LHC upgrades.

Finally, it must be a least mentioned that there is, naively, a contradiction when the Higgs vacuum field is compared to the measured dark energy level. The dark energy comprises, at present, some $74 \%$ of the universe by mass. Then, crudely the dark energy implies, if it is a cosmological constant, a vacuum field related to the critical density $\rho_{c}$. That field is only $2.4 \mathrm{meV}$, while the Higgs field is $174 \mathrm{GeV}$, which is an embarrassing mismatch, Eq. (18), by a factor around $10^{14}$. This naive issue of why the Higgs vacuum field energy is gravitationally inert is a question for 


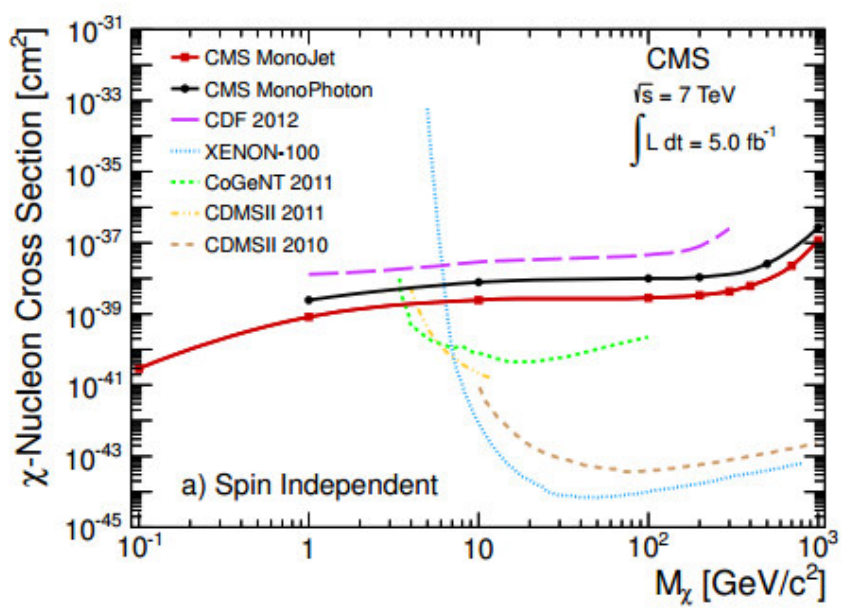

Fig. 55. CMS limit on the spin independent cross-section for a neutralino to scatter off a nucleon as a function of the neutralino mass.

future research and theoretical development

$$
\langle\phi\rangle^{4} \sim \rho_{c}, \quad\langle\phi\rangle \sim 0.0024 \mathrm{eV}, \quad\langle\phi\rangle_{H} \sim 174 \mathrm{GeV} .
$$

\section{Conclusion}

The 20 year search at the LHC for the Higgs boson has been brought to a successful conclusion with the discovery of a narrow state at $125 \mathrm{GeV}$ which couples to vector bosons with approximately the strength of an SM Higgs. Future study will determine the spin and parity of the new state as well as measure the couplings to fermions. An SM Higgs must make the $W+W$ scattering unitary and must have triple and quartic self-couplings which will also be partially tested with data taken in 2012. Further in the future, the mechanism which protects the Higgs mass from high mass scales must be understood as due to SUSY, extra dimensions, compositeness or a mechanism as yet not thought of. There is a rich physics program at the LHC for the next 20 years of data taking and analysis.

\section{Acknowledgments}

The author has used the collective results of the entire CMS collaboration and thanks all his colleagues and co-workers for creating all these beautiful physics analyses. CMS is truly a collaborative team which produces a flood of new physics results.

CMS congratulate our colleagues in the CERN accelerator departments for the extraordinary performance of the LHC accelerator. CMS thank the computing centers in the Worldwide LHC Computing Grid for the creation and excellent performance of the computing infrastructure so essential to our analyses. CMS 
gratefully acknowledge the contributions of the technical staff at CERN and other CMS institutes. CMS also thank the administrative staff at CERN and the other CMS institutes.

Finally CMS acknowledge the support from all the funding agencies that contributed to the construction and the operation of the CMS detector: BMWF and FWF (Austria); FNRS a FWO (Belgium); CNPq, CAPES, FAPERJ and FAPESP (Brazil); MES (Bulgaria); CERN; CAS, MoST and NSFC (China); COLCIENCIAS (Colombia); MSES (Croatia); RPF (Cyprus); MEYS (Czech Republic); MoER, SF0690030s09 and ERDF (Estonia); Academy of Finland, MEC and HIP (Finland); CEA and CNRS/IN2P3 (France); BMBF, DFG and HGF (Germany); GSRT (Greece); OTKA and NKTH (Hungary); DAE and DST (India); IPM (Iran); SFI (Ireland); INFN (Italy); NRF and WCU (Korea); LAS (Lithuania); CINVESTAV, CONACYT, SEP and UASLPFAI (Mexico); MSI (New Zealand); PAEC (Pakistan); MSHE and NSC (Poland); FCT (Portugal); JINR (Armenia, Belarus, Georgia, Ukraine, Uzbekistan); MON, RosAtom, RAS and RFBR (Russia); MSTD (Serbia); SEIDI and CPAN (Spain); Swiss Funding Agencies (Switzerland); NSC (Taipei); TUBITAK and TAEK (Turkey); NASU (Ukraine); STFC (United Kingdom); DOE and NSF (USA).

\section{References}

1. L. D. Landau, Collected Papers (Pergamon Press, Oxford, 1965), p. 546.

2. F. Englert and R. Brout, Phys. Rev. Lett. 13, 321 (1964).

3. P. W. Higgs, Phys. Lett. 12, 132 (1964).

4. P. W. Higgs, Phys. Rev. Lett. 13, 508 (1964).

5. G. S. Guralnik, C. R. Hagen and T. W. B. Kibble, Phys. Rev. Lett. 13, 585 (1964).

6. P. W. Higgs, Phys. Rev. 145, 1156 (1966).

7. T. W. B. Kibble, Phys. Rev. 155, 1554 (1967).

8. S. L. Glashow, Nucl. Phys. 22, 579 (1961).

9. S. Weinberg, Phys. Rev. Lett. 19, 1264 (1967).

10. A. Salam, Weak and electromagnetic interactions, in Elementary Particle Physics: Relativistic Groups and Analyticity, ed. N. Svartholm (Almqvist \& Wiskell, 1968), p. 367.

11. ALEPH, DELPHI, L3, OPAL Collab. and LEP Working Group for Higgs Boson Searches, Phys. Lett. B 565, 61 (2003), arXiv:hep-ex/0306033.

12. ALEPH, CDF, D0, DELPHI, L3, OPAL, SLD Collab., The LEP Electroweak Working Group, The Tevatron Electroweak Working Group, and The SLD Electroweak and Heavy Flavour Groups, Precision electroweak measurements and constraints on the standard model, CERN PH-EP-2010-095 (2010).

13. http://lepewwg.web.cern.ch/LEPEWWG/plots/winter2012/.

14. E. Eichten, I. Hinchliffe, K. Lane and C. Quigg, Rev. Mod. Phys. 56, 4 (1984).

15. J. Gunion, H. Haber, G. Kane and S. Dawson, The Higgs Hunters Guide, Frontiers in Physics (Addison Wesley, 1990).

16. L. Evans (ed.), The Large Hadron Collider: A Marvel of Technology (EPFL Press, 2009).

17. L. Evans and P. Bryant, JINST 03, S08001 (2008).

18. The LHC accelerator, http://public.web.cern.ch/public/en/lhc/lhc-en.html. 
19. G. Jarlskog and D. Rein (eds.), Proc. Large Hadron Collider Workshop, Aachen, Germany, 1990, p. 509, CERN 90-10-V-2/ECFA 90-133-V-2.

20. CMS Collab., Letter of intent by the CMS collaboration for a general purpose detector at the LHC, Technical Report CERN-LHCC-92-03, CERN-LHCC-I-1, CERN (1992).

21. The Compact Muon Solenoid, Technical proposal, CERN/LHCC 94-38, LHCC/P1 December 15, 1994.

22. N. Ellis and T. S. Virdee, Annu. Rev. Nucl. Part. Sci. 44, 609 (1994).

23. D. Green, High $P_{T}$ Physics at Hadron Colliders (Cambridge University Press, 2005).

24. A. Denner et al., Eur. Phys. J. C 71, 1753 (2011).

25. S. Alekhin et al., The PDF4LHC Working Group Interim Report (2011).

26. M. Botje et al., The PDF4LHC Working Group Interim Recommendations (2011).

27. LHC Higgs Cross Section Working Group (S. Dittmaier et al.), Handbook of LHC Higgs Cross Sections: 1. Inclusive Observables (CERN, Geneva, 2011).

28. CMS, the TRIDAS Project, Technical Design Report, Vol. I, CERN/LHCC 2000-38, CMS TDR 6.1, December 15, 2000.

29. M. D. Negra et al., Muon trigger and identification, in Proc. Large Hadron Collider Workshop, eds. G. Jarlskog and D. Rein, Aachen, Germany, 1990, p. 467, CERN 90-10-V-3/ECFA 90-133-V-3.

30. The LHC computing grid, http://public.web.cern.ch/public/en/lhc/Computingen.html.

31. CMS Collab., JINST 3, S08004 (2008).

32. D. Green (ed.), At the Leading Edge, the ATLAS and CMS LHC Experiments (World Scientific, 2010).

33. CMS Collab., JINST 5, T03001 (2010).

34. T. Sjostrand, S. Mrenna and P. Z. Skands, J. High Energy Phys. 05, 026 (2006).

35. S. Gieseke et al., Herwig++ 2.0 Release Note (2006).

36. J. Alwall et al., J. High Energy Phys. 09, 028 (2007).

37. GEANT4 Collab., Nucl. Instrum. Methods A 506, 250 (2003).

38. CMS Detector Performance and Software, Physics Technical Design Report, Vol. I, CERN/LHCC 2006-001, CMS TDR 8.1, February 2, 2006.

39. W. Adam et al., J. Phys. G 31, N9 (2005).

40. S. Baffioni et al., Eur. Phys. J. C 49, 1099 (2007).

41. CMS Collab., JINST 6, 9001 (2011).

42. CMS Collab., JINST 6, 11002 (2011).

43. M. Cacciari, G. P. Salam and G. Soyez, J. High Energy Phys. 04, 063 (2008).

44. CMS Collab., b-jet identification in the CMS experiment, CMS Physics Analysis Summary CMS-PAS-BTV-11-004 (2012).

45. CMS Collab., Particle-flow event reconstruction in CMS and performance for jets, taus, and EmissT, CMS Physics Analysis Summary CMS-PAS-PFT-09-001 (2009).

46. CMS Collab., Commissioning of the particle-flow event reconstruction with the first LHC collisions recorded in the CMS detector, CMS Physics Analysis Summary CMSPAS-PFT-10-001 (2010).

47. M. Cacciari and G. P. Salam, Phys. Lett. B 659, 119 (2008).

48. CMS Collab., JINST 7, P01001 (2011).

49. S. van der Meer, Calibration of the effective beam height in the ISR, Technical Report CERN-ISR-PO-68-31, CERN, Geneva (1968).

50. CMS Collab., Absolute calibration of the luminosity measurement at CMS: Winter 2012 Update, CMS Physics Analysis Summary CMS-PAS-SMP-12-008 (2012). 
51. H. Voss et al., TMVA: Toolkit for multivariate data analysis with ROOT, in XI Int. Workshop on Advanced Computing and Analysis Techniques in Physics Research (2007).

52. T. Junk, Nucl. Instrum. Methods A 434, 435 (1999).

53. A. L. Read, J. Phys. G 28, 2693 (2002).

54. L. Moneta et al., The RooStats project, in 13th Int. Workshop on Advanced Computing and Analysis Techniques in Physics Research (ACAT2010) (2010).

55. CDF and D0 Collab., Phys. Rev. Lett. 104, 061802 (2010).

56. CMS Collab., Search for resonant $t t$ production in lepton plus jets events in $p p$ collisions at $\sqrt{s}=7 \mathrm{TeV}$, arXiv:1209.4397.

57. CMS Collab., Phys. Lett. B 710, 26 (2012), arXiv:1202.1488.

58. ATLAS Collab., Combined search for the standard model Higgs boson in $p p$ collisions at $\sqrt{s}=7 \mathrm{TeV}$ with the ATLAS detector, accepted for publication in Phys. Rev. D, arXiv:1207.0319.

59. ATLAS and CMS Collab., LHC Higgs Combination Group, Procedure for the LHC Higgs boson search combination in Summer 2011, Technical Report ATL-PHYS-PUB 2011-11, CMS NOTE 2011/005 (2011).

60. CMS Collab., Phys. Lett. B 716, 30 (2012).

61. ATLAS Collab., Phys. Lett. B 716, 1 (2012).

62. CDF and D0 Collab., Evidence for a particle produced in association with weak bosons and decaying to a bottom-antibottom quark pair in Higgs boson search at the Tevatron, submitted to Phys. Rev. Lett., arXiv:1207.6436.

63. CDF Collab. (T. Aaltonen et al.), Phys. Rev. Lett. 108, 151803 (2012).

64. C. N. Yang, Phys. Rev. 77, 242 (1950).

65. N Samios, R. Plano, R. Prodell, M. Schwartz and J. Steinberger, Nevis-97, ACC0342 (1962).

66. D. Bisello et al., Nucl. Phys. B 350, 1 (1991).

67. S. Y. Choi et al., Phys. Lett. B 553, 61 (2003).

68. Y. Gao et al., Phys. Rev. D 81, 075022 (2010).

69. CMS Collab., Measurment of the sum of $W W$ and $W Z$ production with $W+$ dijet events in $p p$ collisions at $\sqrt{s}=7 \mathrm{TeV}$, arXiv:1210.7544 [hep-ex].

70. K. Cheung, C. Chiang and T. Yuan, Phys. Rev. D 78, 051701 (2008).

71. CMS Collab., CMS at the high energy frontier, presented to the European Strategy for Particle Physics, Sept. 10-12, Krakow, Poland.

72. S. Dawson, S. Dittmaier and M. Spira, Phys. Rev. D 58, 115012 (1998).

73. A. Djouadi, W. Kilian, M. Muehlleitner and P. M. Zerwas, Eur. Phys. J. C 10, 45 (1999).

74. U. Baur, T. Plehn and D. Rainwater, Phys. Rev. Lett. 89, 151801 (2002).

75. CMS Collab., Search for dark matter and large extra dimensions in monojet events in $p p$ collisions at $\sqrt{s}=7 \mathrm{TeV}$, CMS-EXO-11-059, CERN-PH-EP-2012-168, arXiv:1206.5663. 